Commentary

\title{
Targeting the DNA-PK complex: Its rationale use in cancer and HIV-1 infection
}

\author{
C. Schwartz, O. Rohr, C. Wallet* \\ University of Strasbourg, EA7292, DHPI, IUT Louis Pasteur, Schiltigheim, France
}

\section{A R T I C L E I N F O}

\section{Keywords:}

DNA-PK

DSB repair

Cancer

Virus integration

HIV-1

\begin{abstract}
A B S T R A C T
The DNA-PK complex is the major component of the predominant mechanism of DSB repair in humans. In addition, this complex is involved in many other processes such as DNA recombination, genome maintenance, apoptosis and transcription regulation. Several studies have linked the decrease of the DNA-PK activity with cancer initiation, due to defects in the repair. On another hand, higher DNA-PK expression and activity have been observed in various other tumor cells and have been linked with a decrease of the efficiency of anti-tumor drugs. It has also been shown that DNA-PK is critical for the integration of the HIV-1 DNA into the cell host genome and promotes replication and transcription of the virus.

Targeting this complex makes therefore sense to treat these two pathologies. However, according to the status of HIV-1 replication (active versus latent replication) or to the tumor grade cells (initiation versus metastasis), the way to target this DNA-PK complex might be rather different. In this review, we discuss the importance of DNA-PK complex in two major pathologies i.e. HIV-1 infection and cancer, and the rationale use of therapies aiming to target this complex.
\end{abstract}

\section{Introduction}

Double strand break (DSB) is the most deleterious damage that threats DNA integrity. This frequent damage arises from environmental stress like UV, chemical agents or ionizing radiations (IRs). Although environmental stress does not lead directly to DSB, it produces base damage, mutations and DNA abnormalities. When they are not repaired, lesions cause single strand gaps (SSGs) or distortion in the DNA helix and this mechanical stress eventually leads to DSB. Endogenous stress is also a major cause of DNA damage. Exposure to endogenous reactive oxygen species may provoke DSBs. DSBs may also arise from polymerase operating errors or at stalled replication fork [1].
In developing lymphocytes, DSB is required to initiate V(D)J recombination and Class Switch Recombination (CSR), two essential processes for the development of $\mathrm{T}$ and $\mathrm{B}$ cells, and Immunoglobulins (Igs) production $[2,3]$. However, the consequences of frequent DSB can be dramatic on genome stability. It can lead to DNA and RNA polymerases stalling, uncompleted replication, and chromosomal cross-over and as a general rule to the loss of genetic information.

During evolution, several DNA damage repair pathways have been selected to face the diversity of DNA damages. These repair pathways take part to the global DNA Damage Response (DDR) of cells. The majority of mutations and single base damages due to exogenous stress (UV, chemicals..) or replication error are detected by specific

\footnotetext{
Abbreviations: Alt-NHEJ, alternative NHEJ also called MMEJ; APLF, Aprataxin and PNK-Like Factor; AsiDNA or DT01, Dbait molecule associated with a derivative

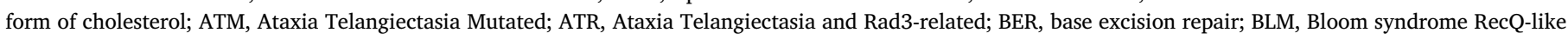

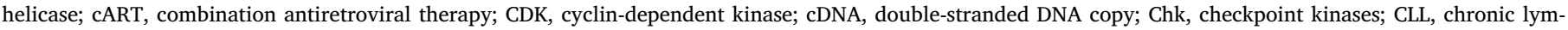

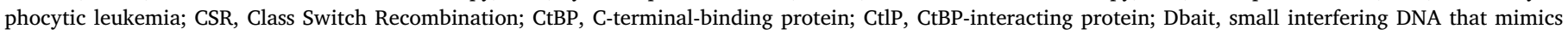

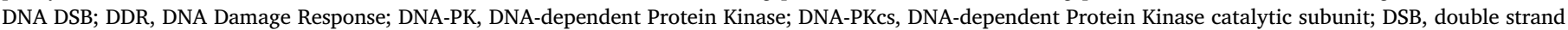

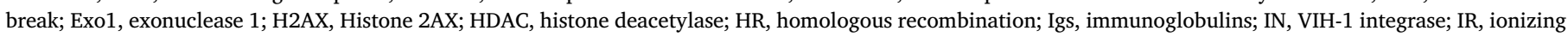

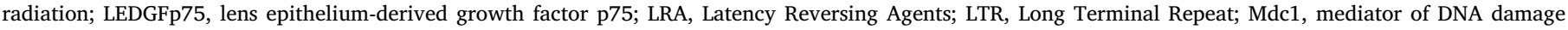

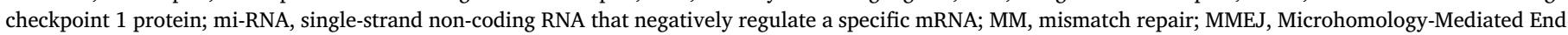

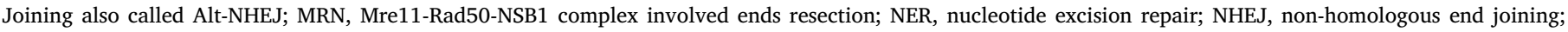

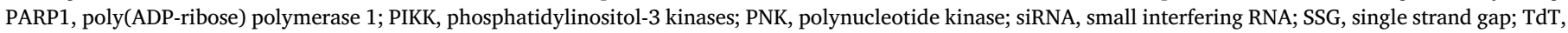

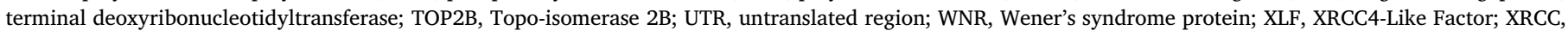
X-ray Repair Cross Complementing

* Corresponding author.

E-mail address: clementine.wallet@unistra.fr (C. Wallet).
} 


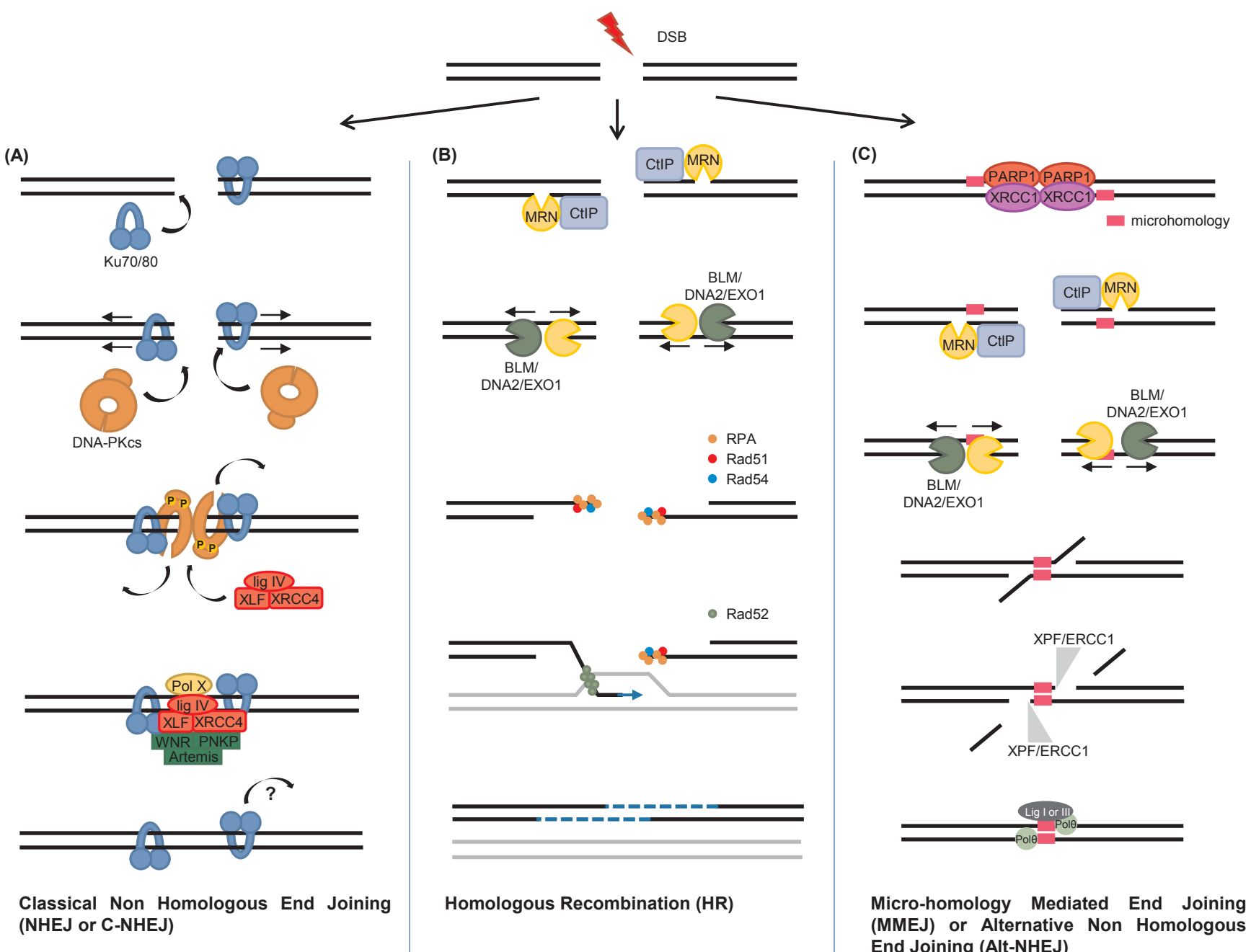

(NHEJ or C-NHEJ) End Joining (Alt-NHEJ)

Fig. 1. Three alternative pathways of DSB repair in eukaryotes. DSBs are formed either by DNA damage agents exposure (e.g. ionizing radiation) or during V(D)J recombination. NHEJ and HR are the two main pathways of DSB repair. NHEJ directly binds two ends of broken DNA regardless of the sequence and with minor DNA resection. This is an easy and fast repair, available at any time of the cycle. HR is a low error prone pathway that needs large DNA resection and the availability of sister chromatid for DNA synthesis. The third pathway is called either MMEJ or Alt-NHEJ and is considered as secondary and highly error prone. In MMEJ, DNA ends are resected while strand invasion and D-loop formation are inhibited. (A) DNA-PK complex assembly at DSB in NHEJ-mediated repair. Ku heterodimer (shown in blue) composed of Ku70 and Ku80, is one of the first to detect DSBs. Ku heterodimer binds rapidly to the ends of the DSB and is responsible for the recruitment of others NHEJ component. DNA-PKcs (shown in orange) is recruited by Ku at the breaks. Ku translocates inward the lesion (indicated by arrows) and allows the DNAPKcs to be placed at the extremities of the break form synapsis between the two broken ends to avoid translocations. Recruitment of XRCC4-DNA ligase allows extensive DNA-PKcs phosphorylation (circles with P) that induces large conformational changes and promotes DNA-PKcs release from the complex. XRCC4-Lig4-XLF seals the ends after an eventual resection step. DSB is repaired with eventual addition or loss of nucleotide. Ku might be removed from the repaired double strand by ubiquitination-driven dissociation. (B) Main steps of HR-mediated DSB repair. The MRN complex (shown in yellow) binds at DSB and initiates the cleavage at the 5' extremity with CtIP (shown in pale blue). DNA is then extensively resected with the help of the ExoI exonuclease, the BLM complex and the Dna2 endonuclease (shown in green). RPA binds to the resulting $3^{\prime}$ overhang ends to stabilize ssDNA. Rad51 and Rad54 also bind to DNA to form the nucleoprotein filament. Recruitment of Rad52 promotes strand invasion in the complementary sequence and stabilizes the forming D-Loop. Then polymerase extends the $3^{\prime}$ overhang extremities (blue arrow). The repair is completed by the resolution of recombination intermediates after elongation. (C) Main steps of MMEJ-mediated DSB repair. PARP1 (shown in red) binds to DSB and with XRCC1 (shown in purple) facilitates the recruitment of resection factors MRN and CtIP. DNA resection is performed with MRN and BLM, EXOI and DNA2 to uncover micro-homologies (pink boxes). The two micro-homologies anneal together and form two non-homologous flaps. This step is inhibited by RPA, which promotes strand invasion and HR-mediated repair. The two flaps are trimmed by the XPF/ERCC1 nuclease (shown in light grey). Gaps are then filled by Pole (shown in light green) and by the ligase I or III (shown in dark grey). (For interpretation of the references to colour in this figure legend, the reader is referred to the web version of this article.)

mechanisms, i.e. base excision repair (BER), nucleotide excision repair (NER) and mismatch repair (MM). The first step of these pathways is cleavage and excision of DNA in order to replace the altered DNA. If uncompleted, the gap blocks replication and eventually causes DSBs [4].

DSB repair is essential to the cell survival and DNA integrity. Three different DSB repair pathways exist; Homologous Recombination (HR) and Non-Homologous End Junction (NHEJ) are predominant while the microhomology-mediated end joining (MMEJ), also named alternative
NHEJ (Alt-NHEJ) is considered as secondary (Fig. 1). HR is initiated by ends resection, which allows single strand invasion in a homologous region and a D-Loop formation. In humans, the Mre11-Rad50-NSB1 (MRN) complex associated with CtBP-interacting protein (CtIP) initiates $5^{\prime}$ nick to create single strand extremities. Subsequently, RAD51 promotes strand invasion in a homologous region, and new DNA is copied from the complementary strand (Fig. 1B). HR is predominant during S and G2 phase. Indeed, proximity of the sister chromatid allows an accessible homologous template, and promote HR. This pathway is 
considered as error-free [5]. On the contrary, NHEJ promotes the ligation of two DNA blunt ends without DNA synthesis. Fast binding of the Ku70/Ku80 heterodimer (Ku) at breaks stabilizes DNA ends and allows recruitment of the DNA-dependent Protein Kinase catalytic subunit (DNA-PKcs) and ligation of the two ends (Fig. 1A). This pathway is faster and completed within $30 \mathrm{~min}$ compared to several hours for HR [6]. However, when multiple breaks occur, NHEJ may induce improper joining between DNA ends and drive chromosomal translocations, depletions or duplications. This error-prone pathway is predominant in humans during G0 and G1 phase but is active in all phases. It is indeed considered that the fast replication of the whole DNA is preferred for transmission. On the other hand, since non-coding regions make up an extensive part of DNA in eukaryotes, there is statistically less chance for errors to occur in a coding region [7]. A third alternative pathway, MMEJ or alt-NHEJ has been recently unveiled. Although this mechanism of repair is considered as highly error-prone, studies showed an important role in DSB repair depending on the biological context. MMEJ promotes ligation by microhomology-mediated annealing of the two ends. The CtIP/MRN complex is recruited by poly (ADP-ribose) polymerase 1 (PARP1) at the DNA breaks, which promote single-strand nick, and extensive resection of ends by the Bloom syndrome RecQ-like helicase (BLM)/exonuclease 1 (EXO1) to expose flanking microhomologies. It results in two single stranded ends flanking the DSB that anneal together by microhomology of 2 to 20 overlapping bases. XPF/ERCC1 nuclease trims non-homologous tails followed by the extension of the strand by the low fidelity DNA polymerase $\theta$ prior ligation by ligase 3 or ligase 1 [8] (Fig. 1C).

In this review we will focus on the canonical NHEJ and on main actors of this complex mechanism. We will discuss the carcinogenic potential of the deregulation of these proteins and how this carcinogenicity can be prevented. We will also discuss the possibility to target this repair key factor to potentiate the IRs effect on the tumor cells to trigger cell death. Finally we will discuss the role of the NHEJ actors in the integration of the virus HIV in the genome.

\section{The central role of DNA-PK in the NHEJ}

NHEJ can be divided in several steps (Fig. 1A): 1) recognition and binding of $\mathrm{Ku}$ at each ends of the DSB following by the assembly of the DNA-PK at the break points and bridging; 2) phosphorylation of the DNA ends by DNA-PKcs; 3) ligation of the ends by DNA ligase IV/ XRCC4 complex; 4) complex dissociation.

\subsection{DSB detection and complex assembly}

$\mathrm{Ku}$ is the first protein to bind to the damaged DNA. Ku70 (73 kD) and Ku80 ( $86 \mathrm{kD})$ both contain a "core" domain that bind to DNA. When Ku70 and Ku80 heterodimerize, they form a ring-like structure around approximately 20 base pair of double stranded DNA and strongly bind at DSB in a sequence-independent manner [9]. Early recruitment of $\mathrm{Ku}$ to breaks (within $3 \mathrm{~min}$ ) can be explained by the high abundance of $\mathrm{Ku}$ in cells [10]. Ku shows high affinity for double strand DNA, contrary to single-stranded DNA, and only binds to the ends that has not been resected [9]. The primordial role of $\mathrm{Ku}$ in damage sensing will be discussed later. The heterodimer allows ends stabilization and protects DNA extremities from any extensive resection that promotes HR. Moreover, it was shown that MMEJ operates only if $\mathrm{Ku}$ is removed from the ends suggesting that $\mathrm{Ku}$ inhibits this highly error-prone pathway. The Ku heterodimer also acts as a scaffold for the NHEJ, and recruits necessary factors for repair. Among these factors, DNA-PKcs is rapidly recruited at break points. DNA-PKcs belongs to the Ser/Thrprotein kinase family of phosphatidylinositol-3 kinases (PIKK) that also includes Ataxia Telangiectasia Mutated (ATM) and Ataxia Telangiectasia and Rad3-related (ATR), two proteins involved in the DNA damage sensing. Two units of DNA-PKcs interact with two units of Ku70/80 to form the DNA-PK complex. Binding of DNA-PKcs to Ku and to double-stranded DNA activates the kinase activity and promotes the translocation of $\mathrm{Ku}$ heterodimers toward the break to allow the binding of others enzymes. The presence of DNA-PK at each side of the break allows bridge formation between the two ends in a synapsis, and reduces the chance of improper joining. The catalytic activity of DNA-PK is required for the repair to be accurate. It has been shown that DNAPK, phosphorylates every actors (Ku70, Ku80, XRCC4, XLF, Artemis, PNKP and DNA-PKcs) of NHEJ, but only auto-phosphorylation is essential. Several auto-phosphorylations seem to promote the bridge of termini, eventual DNA ends processing and the recruitment of XRCC4Lig4, but theses mechanisms are still unclear. Auto-phosphorylation of Serine 2056 and more largely in other sites of the cluster called PQR is considered as an activation indicator of DNA-PK for repair in cells [11].

\subsection{DNA resection}

Depending on the damage, DNA ends are not always blunt at breaks. In particular IR damages may generate intricate non-ligatable ends that may differ from one break to another, like overhanging ends, 3'-phosphate groups or $5^{\prime}$-hydroxyl groups. In addition, cell processes involved in $\mathrm{V}(\mathrm{D}) \mathrm{J}$ recombination creates secondary structure DNA elements like hairpin that has to be treated prior ligation. Several proteins are involved in the process of DNA ends in order to ligate the termini and are considered as secondary to the NHEJ: Artemis, Terminal deoxyribonucleotidyltransferase (TdT), DNA polymerase $\mu$ and $\lambda$ (Pol $\mu$ and Pol $\lambda$ ), polynucleotide kinase (PNK), and possibly the Wener's syndrome protein (WNR) and Aprataxin and PNK-Like Factor (APLF). Resection may lead to the loss or insertion of one or several nucleotides and increases the risk of errors during repair. In addition to its protective function at breaks, $\mathrm{Ku}$ is important in the ends processing by direct recruitment of processing factors like WRN, TdT, Pol $\mu$, Pol $\lambda$ and APLF.

Artemis and TdT have functions in the DNA ends processing during V(D)J recombination. While Artemis seems to have limited role in the DSB repair, its presence is essential at DNA hairpin occurring during V (D)J recombination. TdT is expressed only in lymphocytes and its role in nucleotide addition between recombining coding sequences has been described in V(D)J recombination. Pol $\mu$ and Pol $\lambda$ are members of DNA pol X family and have gap-filling activities. They are recruited at DSB by Ku associated with the XRCC4/Lig4 complex and allow the filling of gaps coming from ends processing prior repair. PNK, WNR and APLF possess exonuclease activities and are good candidates for various ends processing during NHEJ mediated repair. Steps of DNA ends processing are susceptible to insert or delete one or several nucleotides thus reinforcing the tendency of NHEJ to be error-prone [12,13].

\subsection{Ligation and complex dissociation}

Once the break is stabilized by DNA-PK and the ends are processed, ligation can occur. During ligation XRCC4/Lig4 is recruited by $\mathrm{Ku}$ through direct interaction. Phosphorylation of XRCC4/Lig4 by DNA-PK stabilizes the NHEJ complex and strengthens connections between NHEJ factors. In addition, the XRCC4-like factor (XLF) and eventually APLF are also recruited by $\mathrm{Ku}$. These interacting proteins potentiate ligation of both DNA strands by XRCC4/Lig4, possibly by adenylation of Lig4. XLF is also phosphorylated by DNA-PK [14]. Several lines of evidences show that DNA-PK is destabilized by auto-phosphorylation at a specific cluster called ABCDE and promotes DNA-PKcs dissociation from the complex [15].

Repair is complete after ligation, but $\mathrm{Ku}$ is trapped around the double strand DNA molecule because of its ring-like structure. Several hypotheses have been introduced to explain the escape mechanism of $\mathrm{Ku}$. The most accepted theory for Ku removal in human cells is the ubiquitin-mediated degradation of $\mathrm{Ku}[16,17]$. 


\subsection{DSB sensing and chromatin compaction}

DSB repair occurs at different steps of the cell cycle. The chromatin has to be unpacked to allow access of the DNA repair machinery to damages. An efficient mechanism of DSB sensing exists, which signals damage and amplifies DDR. The MRN complex is one of the first complex to be localized at DSB. It was shown that MRN recruits and activates ATM at breaks. ATM phosphorylates H2AX, a member of H2A histone family, at Serine 139. The phosphorylated form of H2AX, $\gamma \mathrm{H} 2 \mathrm{AX}$, is a specific marker of DSBs [18]. Alternatively, Ku recruits DNA-PKcs which phosphorylates $\mathrm{H} 2 \mathrm{AX}$ at breaks and promote a cascade of phosphorylation. Subsequent mono-ubiquitination of $\gamma \mathrm{H} 2 \mathrm{AX}$ allows cells to distinguish between damage that can be repaired and chromatin damages eliciting apoptosis [19]. Furthermore, $\gamma \mathrm{H} 2 \mathrm{AX}$ recruits mediator of DNA damage checkpoint 1 protein (Mdc1). $\gamma \mathrm{H} 2 \mathrm{AX}$ together with Mdc1 enhance a self-reinforcing mechanism that promotes $\mathrm{H} 2 \mathrm{AX}$ phosphorylation to expand and spread $\gamma \mathrm{H} 2 \mathrm{AX}$ formation. Histone phosphorylation promotes chromatin decompaction and facilitates DNA repair proteins to access breaks. $\gamma \mathrm{H} 2 \mathrm{AX}$ together with Mdc1 also activate ATM that phosphorylates ATR, and 53BP1 and BRCA1 in cascade. These two tumor suppressors are essential regulators of DSB repair pathway choice. 53BP1 and BRCA1 antagonize the response to DSB. 53BP1 favors NHEJ by repressing resection in G1 phase, while BRCA1 together with CtIP enhance 53BP1 removal and promote HR in G1/S transition. Alternatively, phosphorylation of p53 and checkpoint kinases (Chk1/2) by ATM/ATR promotes cell cycle arrest and/or apoptosis [20]. Ku seems to repress ATM activation of ATR, in order to signal the damage to HR pathway [16].

\subsection{Regulators of NHEJ: the repair pathway choice}

Cells possess an efficient sensing system to track DSBs. Since several DSB repair pathways co-exist, the question of the DSB repair choice has been largely addressed [21]. Depending on the phase of the cell cycle, or damage severity, the pathway of repair has to be an adaptive response to allow the multiplication of cells but also the transmission of an entire and non-mutated genetic material. Several factors like the chromatin condensation state, the availability of a sister chromatid, and the state of DNA ends, promote or inhibit HR or NHEJ in order to favor one pathway toward another one. Some factors interplay at critical points of the cycle to make a checkpoint on the DNA integrity and state of replication. Depending of the severity of the damages and their frequencies, cell cycle is arrested to give time for the repair and avoid replication or mitotic catastrophe. In case of extensive damages, several factors trigger apoptosis to avoid multiplication and the spread of potential mutation [22].

The presence of several checkpoints in the cell cycle allows the control of DNA integrity. A first ATM, Chk2 and p53 regulate the G1/S checkpoint prior the replication. A second checkpoint during the $\mathrm{S}$ phase allow the delay of replication start to give extra-time for the repair of any damage and is regulated by ATR, DNA-PK, WEE1 and Chk1. This checkpoint avoids under-replicated sequences of DNA. The last checkpoint in the G2/M phase is the most critical point to avoid mitotic catastrophe caused by damaged DNA or under-replicated DNA. Chk1, MYT1 and WEE1 activities can delay the mitotic entry of the cell. If DNA damages are too dramatic to be repaired, apoptosis is driven by p53 and avoid the proliferation of potential mutated cells [23].

Because of the abundance of DNA-PKcs and Ku heterodimer in human cells, it has been postulated that NHEJ is the default pathway to repair DSB. In addition it has been shown that NHEJ is active in every cell cycle [11]. However, several factors influence the pathway choice.

The two well-known tumor suppressor p53 and BRCA1, have a key role in DSB repair pathway choice. p53 is an important transcription factor for apoptosis, DNA repair and cell cycle arrest. Its role in environmental stress adaptation and metabolic regulation has been largely described. p53 can either promote cell cycle arrest at G1 phase and
NHEJ, or apoptosis if damage are too serious or if cells are in S/G2 phase [24]. The precise function of BRCA1 is still unclear but it could block DNA-PKcs auto-phosphorylation and promotes HR in S/G2 phase [25].

In S and G2 phase, extensive resection is stimulated by cyclin-dependent kinase (CDK) activity that phosphorylates CtIP, and MRN complex. They promote single strand DNA that is less susceptible to bind with $\mathrm{Ku}$, and favor HR towards NHEJ. CDK also downregulates 53BP1 in S/G2 phase. In G1/M phase, 53BP1 inhibits BRCA1 and limits end resection to favor NHEJ [21]. PARP1 directly interacts with $\mathrm{Ku}$ at breaks and decreases the affinity of Ku for DNA that has the consequence to favor the MMEJ repair toward NHEJ [21].

\section{DNA damage repair and cancer}

Cancer has been described as a disease of DNA repair, pointing out the strong link between DNA repair and cancer. Studies of pre-cancerous lesions, allowed the drawing of a model for carcinogenesis. Comparison between normal cells and premalignant human tumors of urinary bladder, breast, lung and colon, have highlighted an increase of DDR activity but also an increase of the senescence and apoptosis index, early in tumorigenesis. There is indeed in these cells an increase of DSBs due to replication stress that drives genomic instability. Replication stress is generated by oncogenes and is due to checkpoint, cell cycle or DNA repair defects that lead to replication fork collapse and finally to DSBs. On the other hand, the high rate of senescence and apoptosis driven by the tumor suppressor p53 is a barrier to cancer. However, genomic instability in precancerous cells can lead to further mutations that confer cancer properties to cells. These mutations often affect p53 and abolish apoptosis in cancer cells but they can also affect DNA repair, cell cycle checkpoint and other apoptotic protein activities $[26,27]$.

Sequencing of cancerous cells DNA showed an increased level of endogenous DNA damages compare to normal cells [28]. This extensive DNA rearrangement in tumors point out the defect and abnormality of DNA damage repair in cancerous cells. Indeed, several lines of evidence show that almost all cancerous cell present DNA repair defects [29].

Normal cells ensure DNA integrity at various cell cycle checkpoints. Cell cycle checkpoints are tightly related to DNA repair as several repair factors play the role of checkpoint proteins, or are recruited by them. Cell cycle checkpoint protein deregulations can result in a high replication rate that characterizes cancerous cells. In fact, the deleterious effect of cancerous cells comes from their capacity to cycle often. Indeed, DNA repair key factors or cell cycle checkpoint proteins defects predispose for cancer. For example deficiencies in factors that promote $\mathrm{HR}$, like BRCA1 or BRCA2, predispose for breast and ovarian cancer, and mutations in the cell cycle factor ATM, increase malignancies incidences including acute leukemia [23].

If tumors cells are deficient in one of the DNA damage repair pathway, they paradoxically strongly rely on other DNA damage repair to survive. As one of the DNA damage repair pathway is deficient in cancerous cells, they are in fact "addicted" to an alternative pathway of repair, which is often causing genetic instability. In addition, the high replication rate increase genetic instability and make them even more dependent to DNA repair [30]. Moreover, it has been shown that an overactive DNA damage repair in cancerous cells is associated with poor prognosis for patient. An over-efficient DNA damage repair provokes resistance to DNA damaging agent and is a barrier to treatment responsivity of malignancies [31].

DNA damage repair factors are crucial for several stage of cancer. If over-activation of DNA damage repair increases the malignancy and resistance of cancer cells, both under- and over-activation of DNA damage repair lead to tumorigenicity in normal cells. Therefore, all DNA damage repair complexes constitute a promising source of anticancer drug targets. In fact maintaining equilibrium between DNA repair pathways could prevent carcinogenesis, while inhibiting DNA repair in 
tumor could improve DNA-damaging treatments.

\subsection{NHEJ and carcinogenesis}

The loss of a NHEJ core factors has been rarely observed in genetic disease. Genetic defects of DNA-PKcs, LIG4 and XRCC4 has been observed and were associated with a diversity of serious global symptoms like severe SCID, growth retardation, microcephaly, immunodeficiency and/or radio-sensitivity. These data showed the importance of fast repair by NHEJ during G1/S phase, even if other pathways of DSB repair exist. NHEJ is also necessary for development of innate immunity, namely $\mathrm{V}(\mathrm{D}) \mathrm{J}$ recombination and CSR [31].

Observations in cell culture or in mice have shown that mutation or loss of one of the core NHEJ factors (Ku70, Ku80, DNA-PKcs, Lig4, XRCC4 or XLF), induced chromosomal exchanges and high sensitivity to toxic agents causing DSBs. Furthermore, some studies reported a downregulation of Ku70, Ku80 or DNA-PKcs in tumor tissues of several types of cancer which could be linked to chromosomal instability. In addition, a reduced activity of DNA-PKcs resulted in an increased risk for lung cancer [32].

Dysfunctional NHEJ may have serious consequences on genetic stability. Inefficient NHEJ may be responsible for the ligation of two ends of two different chromosomes. Moreover, cells with NHEJ defects are more sensitive to DNA damaging agents and accumulation of DSBs or mutations can be dramatic for DNA integrity. If NHEJ is lost, repair may occur either by HR in a long time range, or by the highly errorprone MMEJ when sister chromatid is not available. This leads to an accumulation of DSBs or to large mutations driven by MMEJ. Thus deregulation or dysfunction of one of the core factor of NHEJ might enhance the risk of cancer [26].

Surprisingly, upregulation of NHEJ activity by overexpression or activation of Ku70, Ku80 and DNA-PKcs has also deleterious effect on chromosomal stability. A line of evidence shows that Ku protein expression is increased in tumor cells versus normal cells in gastric and colorectal cancer $[33,34]$. The same correlation has been established between DNA-PKcs overexpression and carcinogenesis in prostate, lung, colorectal, esophageal and gastric cancer [31]. It can be postulated that predominance of an error-prone pathway such as NHEJ, may inhibit the accurate HR pathway and drives genomic instability.

Finally, a number of studies characterized various single nucleotide polymorphisms (SNP) of Ku80 and Ku70 and one in DNA-PKcs which were related to several types of cancer susceptibilities. Surprisingly, another DNA-PKcs variant has been shown to protect against breast and lung cancer. This strengthens the idea that NHEJ has a strong influence in tumorigenicity [29].

In normal cells, tumorigenic effect of both downregulation or upregulation of NHEJ core factors suggest that a finely controlled balance exists between the various repair mechanisms, thus inhibiting cancer formation [31]. Indeed it could be deleterious to over or under activate the NHEJ pathway. A promising approach would be to find biomarkers that detect early tumors. The measurement of DSBs, by a specific marker such as $\gamma \mathrm{H} 2 \mathrm{AX}$, might be used to detect precancerous lesion. Interestingly, synchronous measurement of $\gamma \mathrm{H} 2 \mathrm{AX}$ and p53 might be an efficient tool to diagnose early tumors. In addition, measurement of $\gamma \mathrm{H} 2 \mathrm{AX}$ and DNA-PK could be useful to detect discrepancies in the NHEJ process $[29,35]$.

\subsection{NHEJ in cancer progression}

NHEJ appears to be one of the guardians of genome integrity in vertebrates, but this capacity is turned into a threat in the context of cancer. Many studies have shown a correlation between the increase of $\mathrm{Ku}$ and DNA-PKcs expressions and a high tumor proliferation rate, metastasis and poor prognosis of survival [31].

Several studies showed better therapy responsiveness in NHEJ defective tumors of ovarian cancer tissues, cervical carcinomas and hypopharynx cancer [31]. In these studies, tumor cells with lower expression of $\mathrm{Ku}$ or DNA-PKcs were more sensitive to DNA-damage. Conversely, increased $\mathrm{Ku}$ expression increased resistance to DNA-damaging agents of rectal carcinoma and lung adenocarcinoma while increased DNA-PKcs expression correlated with radiation resistance in lung cancer lines, glioma, cervical and breast cancers, and in B-cell chronic lymphocytic leukemia (CLL). An increase of the NHEJ activity in tumor cells is associated with tumor proliferation and malignancies which promotes DNA damaging agent resistance.

DNA-PKcs is regulating the replication checkpoint in S phase but also play a role in mitosis [14]. These functions of DNA-PKcs, in addition with DNA repair can explain why deregulation and over-expression of DNA-PKcs enhance malignancy and resistance to treatment. On another hand, $\mathrm{Ku}$ has been shown to inhibit the BAX-mediated apoptosis in vitro. This potential anti-apoptotic activity of Ku could promote tumor cell proliferation in tissues over-expressing $\mathrm{Ku}[16,36]$. Finally, it was postulated that an efficient NHEJ repair allows the survival of cancerous cells and promotes cell proliferation [31]. Since NHEJ strongly influences cancer malignancy and therapy responsiveness, NHEJ proteins constitute potential anticancer drug targets.

Hallmarks of cancer are genetic instability, dependency of at least one of the DNA damage repair pathways, escape on apoptosis and proliferation. These features constitute a weakness for malignant cells. Because of the complexity of cancer, it is difficult to target specifically malignant cells [23]. Cancerous cells present a genetic instability and accumulate more DNA damages than normal cells. This trait arises from the defect of one of the DNA-damage repair pathways that characterizes most of cancerous cell types. In these cells, an alternative pathway of DNA damage repair is over-activated to allow cell survival. This DNA damage repair pathway dependency might be used to develop original treatments. As a consequence, targeting DDR actors is investigated in the aim to find tumor treatments [30].

In aggressive tumors, cancerous cells can be radio- or chemo-resistant. Since treatments in this later case have to be more intense, the specificity has to be even higher to prevent severe side effects. One way to get such specificity is to increase the susceptibility of malignant cells to IRs and chemotherapies through the inhibition of the DNA damage repair. Many studies showed the link between several resistances and an increase in the expression of Ku70, Ku80 and DNA-PKcs, making sense to a correlation of the inhibition of NHEJ and the susceptibility of malignant cells to current treatments [31].

\subsection{Targeting DNA-PK with inhibiting molecules}

Numerous inhibitors have been investigated recently. One strategy is to inhibit DNA-PKcs by targeting the ATP-binding pocket with small molecules. DNA-PKcs is included in the PIKK family, with ATR and ATM. PIKK proteins differs from classical protein kinase and belong to the subgroup PI3K. Initial studies used wortmannin and LY294002 as DNA-PKcs inhibitors. However, these two drug families are global PI3K inhibitors and not specific to DNA-PKcs. The spectrum of inhibition of these drugs is large and even if they could sensitize cancerous cells to chemotherapies, studies showed important cytotoxic effect on normal cells. A new generation of selective DNA-PKcs inhibitors, which are derivatives of the small molecule LY294002, has been developed recently. These derivative molecules (NU7441, NU7026, IC86621 and IC87361) enhanced tumor and CLL cells susceptibility to DNA-damaging agents in preclinical in vitro and in vivo studies. Vanillin is another selective inhibitor of DNA-PKcs which possesses an anti-carcinogenic activity. Several cancer cell lines when treated with Vanillin were more sensitive to chemicals but not to radiotherapy [37]. The most promising inhibitor CC-115 is currently in phase I clinical trial. This molecule inhibits both DNA-PK and mTOR kinase. CC-115 has been tested in vitro on a wide variety of cancer cell lines comprising lymphomas, leukemias, hepatocellular carcinoma, breast, lung, head and neck cancer cell-lines. These studies suggest that CC-115 is efficient when 


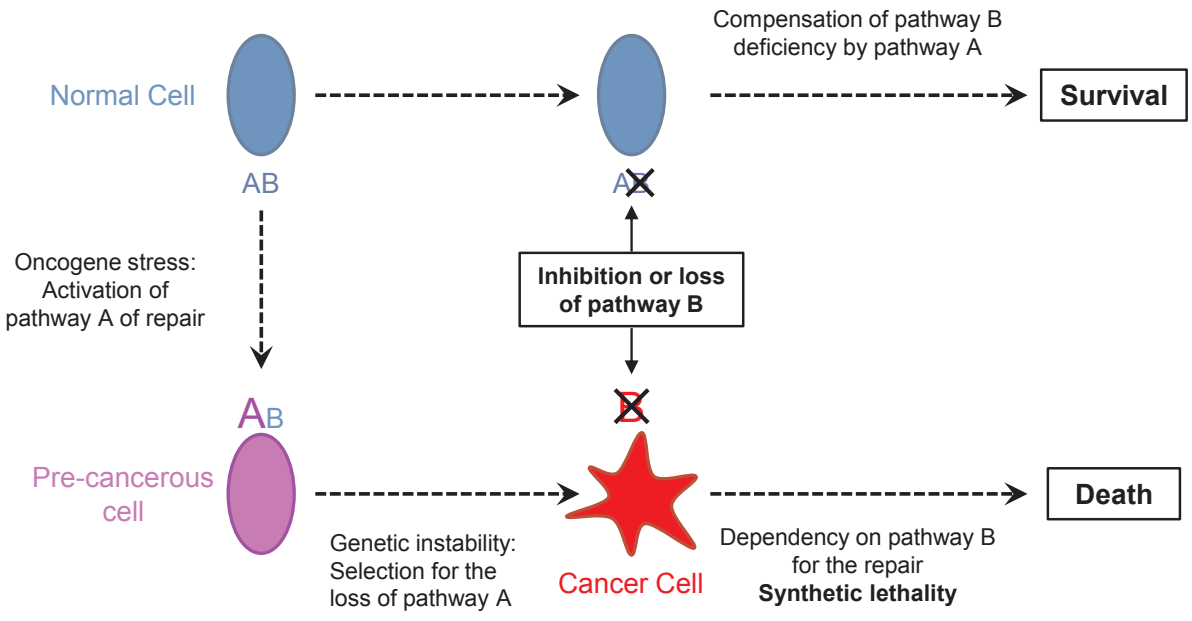

Fig. 2. Use of synthetic lethality to target cancer cells deficient in a repair pathway. Normal cells (shown in blue) possess a whole functional DDR. They can compensate the loss or inhibition of an individual repair pathway. In pre-cancerous cells (shown in purple), oncogene activates DDR. This leads to genetic instability and to the loss of one repair pathway in cancer cells (shown in red). In these cells, inhibition of remaining pathways induce death through the process called synthetic lethality. (For interpretation of the references to colour in this figure legend, the reader is referred to the web version of this article.) used in monotherapy in ATM deficient cells, or in combination of other DNA-damaging agents in some cancers [38].

Another molecule, Dbait, has been tested in phase I clinical trial. Dbait is composed of small double strand DNA molecule that mimics DSB and binds DNA-PK and PARP1. As a consequence, these two crucial repair factors are not available for DSB repair in presence of this molecule. Dbait has been associated with a derivative form of cholesterol, to improve the entry of this molecule in cells. This pharmacological form called AsiDNA (or DT01), has been tested in phase I clinical trial on melanoma in association with radiotherapy. Two studies show the selective increase of cancerous cells sensitization [39,40]. Preclinical studies showed a chemo-sensitization of metastatic colorectal and liver cancers with AsiDNA [41]. In addition, synergy between AsiDNA and conventional cancer therapies (etoposide, cyclophosphamide and radiotherapy) has been shown in 8 out of 10 lines of lymphoma and leukemia cell line. Moreover, AsiDNA increases DNA-damaging agent sensitivity of tumor cells without affecting normal hematological white blood cells [42]. These results are promising and AsiDNA is currently tested on phase I clinical trial as a monotherapy in advanced solid tumor.

The use of micro-RNAs targeting DNA-PKcs and by extension DDR factors could enhance anti-cancer therapies (reviewed in [43]). miRNAs are single-strand non-coding RNA that negatively regulate translation and destabilize a specific mRNA, by binding to the $3^{\prime}$ untranslated region (UTR) of the targeted gene. This results in an inhibition of transcription and in a decrease of the expression of the targeted protein. mi-RNAs have biological functions in cellular stress responses and their expression profiles drastically change during carcinogenesis. Dysregulation of mi-RNA expression in tumor cells can negatively regulates tumor suppressor expression or promote oncogene expressions. Several mi-RNA targeting DDR genes have been identified. The use of synthetic mi-RNA that mimics cellular mi-RNA is interesting to modulate the gene expression involved in tumor persistence. The main advantage to use mi-RNA rather than small-interfering RNAs (siRNAs), which is an established technology for robust gene silencing, is the natural presence of miRNA in cells. Side effects due to off-target are minimized when using mi-RNA rather than siRNAs that are not naturally present in mammal's cells [43]. mi-RNA 101 overexpression has been shown to downregulate ATM and DNA-PKcs and to sensitize tumor xenograft to IRs and pancreatic cancer cells to gemcitabine $[44,45]$. Another study used a mi-RNA $488-3 p$ to sensitize in vitro malignant melanoma cells to cisplatin by targeting the PRKDC gene coding for DNA-PKcs [46]. This strategy of inhibition is still far from clinical application. In these studies, cancerous cells were transfected with lipofectamine. Entry of mi-RNA mimics into the cell and into the nucleus still remains to be improved.

Until recently, specific inhibitors of Ku heterodimers were not available. The ring-like structure of the Ku heterodimers and its strong affinity to broken DNA make difficult the access for their inhibitors. But a small molecule inhibitor of Ku has recently been found by identification of a putative binding pocket in Ku structure [47]. Targeting Ku70 by small interfering RNA has been shown to enhance cancer cell response to topoisomerase II inhibitor and radiation [48]. But these experiments have been performed on cultured cells and there is still a lot of development and improvement before clinical trials.

\subsection{Moving forward to new therapies}

\subsubsection{Use of synthetic lethality in the perspective of monotherapy}

Synthetic lethality is a concept that is promising regarding cancer therapies. Synthetic lethality has been first defined by the lethality of two or more combined mutations when single mutation is viable. This concept has been explored in cancer with DNA damage repair pathways. The concept relies on bypassing one DNA damage repair pathway by using systemic therapy. In normal cells, an alternative DNA damage repair is active and can repair damages. While cancerous cells often lack one of the DNA damage repair pathway. Ideally, this defect in two DNA damage repair pathways would be lethal for cancerous cell (Fig. 2). The aim of using DDR inhibitors as a monotherapy is to reduce side effect due to chemotherapies or IRs $[29,30]$. The best proof of concept is the synthetic lethality between BRCA1 mutation with PARP1 inhibition. Some experiments have shown that HR-deficient xenograft tumor cells (deficient in BRCA1 or 2) were killed by PARP1 inhibitors, in concentration that were non-toxic for normal cells and surrounding tissues $[49,50]$. This lethality can be explained by the lack of PARP1dependent SSG repair, combined with the impossibility to resolve stalled replication fork by recombination via HR. Olaparib has been the first PARP1 inhibitor approved for the market in BRCA-associated breast and ovarian cancer. Six other PARP1 inhibitors are in clinical development phase as monotherapy or in combination with DNA-damaging agent $[23,30]$.

As NHEJ is complementary with $\mathrm{HR}$, it makes sense to investigate synthetic lethality of BRCA deficiency that impaired HR, and DNA-PK inhibition. Since the concept of synthetic lethality is attractive, many difficulties have been encountered. Indeed, resistance has been observed in BRCA2-negative breast cancer. Under PARP1 inhibition selective pressure, a second mutation can lead to the re-expression of BRCA2 and restore the ability of cancer cells to repair and to proliferate [51]. In addition HR can be restored in BRCA-deficient cells by the deletion of DNA-PKcs or 53BP1, a factor promoting NHEJ [52,53]. This suggests that inhibition of NHEJ in HR deficient cells could overcome resistances.

One study has shown the cisplatin sensitization of a breast cancer germ line BRCA1-deficient to the DNA-PKcs inhibitor NU7441 [54]. 
However, this effect was not as pronounced as with the use of PARP1 inhibitors. Indeed it has been pointed out a deficiency of BER (responsible for single gap repair) in the BRCA1-deficient cells used for the study which raise the idea of a synthetic lethality between BER and NHEJ factors.

Another synthetic lethality has been unveiled between a protein involved in mismatch repair, MSH3 and DNA-PKcs. Dietlein and colleagues have actually shown that MSH3 deficient cells have a defect in HR repair, explaining the strong lethality of DNA-PKcs inhibitor in these cells. They also suggest a potential synthetic lethal interaction between that BRCA1, BRCA2, ATM, Rad50 and DNA-PKcs [55].

Jiang and colleagues have shown the addiction to DNA-PKcs of ATM-deficient cancer cells, suggesting synthetic lethality between these two kinases [56]. ATM is important in DNA DSB sensing and is a G1/S checkpoint factor that act as a binary switch to modulate p53-induced apoptosis. In normal cells, ATM together with Chk2 and p53 contribute to induce apoptosis. In the presence of a functional p53, ATM suppression downregulates apoptosis and promotes cell-cycle arrest in G1 phase followed by NHEJ repair using DNA-PKcs. These ATM-deficient cells are resistant to DNA-damaging chemotherapies. Jiang and colleagues have shown that DNA-PKcs knock-down or inhibition sensitizes ATM-deficient tumors cells to genotoxic agents. However treatment has to be improved regarding the type of tumor. Indeed, in cells deficient in p53 which possess a functional ATM, apoptosis is reduced and a robust checkpoint is established by the action of ATM on cell cycle arrest. As a consequence ATM promotes HR directed repair and renders these cells resistant to DNA-damaging agents. In this latter case, inhibition of DNAPKcs is inadequate.

Several cell cycle checkpoint inhibitors are being tested to develop monotherapies. The S-phase checkpoint allows the delay of replication origin firing for the repair. It is a critical step to avoid under-replicated DNA, or more dramatically, stalled replication fork collapse and replication catastrophe [57]. Buisson and colleagues have shown that DNA-PKcs-Chk1 is an alternative pathway to the ATR-Chk1 pathway, involved in cell-cycle arrest during the S-phase. Indeed, DNA-PKcs with Chk1 suppress initiation of replication in the presence of ssDNA. Inhibiting Chk1 impairs the two alternative pathways and is thus more efficient than ATR inhibition to suppress the checkpoint and provoke replication catastrophe in cancerous cells. However, DNA-PKcs inhibitor could be used to deactivate this checkpoint in various cancers with a lower ATR activity or higher replication stress [58].

All these strategies put in light the importance to tailor treatment to every single patient and tumor type. In fact, monotherapies are really promising treatment to target cancer with a minimum of side effects. But the genetic background of tumors should be defined in order to know which factor would be the most efficient to target. As mentioned in this paragraph, targeting DNA-PKcs in non-adapted genetic background could be inefficient for example in p53 deficient and ATM proficient cells. It could also drive resistance by reverse mutation as described with reversion of BRCA2 mutation.

In fact the main problem for the development of new efficient synthetic lethal treatments for cancer is the synthetic lethal penetrance. Indeed it appears that synthetic lethal effect of a pair of gene deficiencies depends on the genetic background of the cells. In the case of soft synthetic lethality, the synthetic lethality interaction is easily abrogated by changes in additional genes. In cancer cells, genomic instability can easily drive further mutations. In this context, soft synthetic lethality is not an ideal cancer therapy compare to hard synthetic lethality [59]. A lot of effort is made to find new synthetic lethal interactions and to define new targets. Advances in next-generation sequencing allow genome wide screening approaches by using RNAi or CRISPR-cas9. These strategies are promising to perform large-scale synthetic lethality screens and find new anticancer targets [60].

\subsubsection{NHEJ inhibition in combination with DNA damaging agent}

Until now, main treatments of cancer after surgery are radiations and chemotherapies. First of all, IRs are used to kill cancer cells. They generate DSBs and SSG directly in the DNA molecule or through the ionization of water. Alternatively, several cytotoxic compounds are used in chemotherapies. Among them, alkylating agents like cisplatin, topoisomerase inhibitors and cytotoxic antibiotics like bleomycin act as DNA-damaging agents by a diversity of mechanisms that finally create DSBs. High replication rate and DNA-damage repair dysfunction sensitize cancerous cells in a greater extent than normal tissues. These two kinds of treatments lead to the death of cancerous cells but have of course side effect since they also affect normal cells in a lower intensity. Moreover, naturally proliferating tissues like bone marrow, gastro-intestinal tract, liver and hair follicles are also sensitive to chemotherapies. In resistant tumors, the treatment dose necessary to kill tumor cells is limited by the normal cells cytotoxicity. A line of evidence correlates cancer resistance or sensitization with dysregulated expression of DDR factors. For example, sensitivity of CLL to cytotoxic agent is correlated with an increase of DNA-PKcs activity [61]. Synthetic lethality is not always sufficient to kill cells and combination with IRs could really improve the therapeutic index. It could allow a decrease of the IR dose and of collateral damages.

Several DNA-PKcs inhibitors have been tested in vivo in preclinical test and have shown good results for the radio-sensitization of tumor cells [37]. One clinical study is ongoing on advanced solid tumors and CLL. This approach is especially interesting to kill aggressive radio-resistant cells.

Due to systemic delivery of drugs and overlapping toxicities with DDR drugs, combination of DNA-PKcs inhibitors with DNA-damageinduced chemotherapies happened to be more challenging. Despite good preclinical results of sensitization of tumor cells, clinical tests have been less successful and have shown toxicity in normal cells. Treatment dose, timing and frequency need further investigation to improve the therapeutic index.

Finally, it would be interesting to define genetic background patterns of resistant and sensitive tumors in order to improve radio and chemotherapy protocols. This would allow a tailoring of treatment to every single patient and adapt the dose of cytotoxic treatments regarding the tumor resistance. The spread of high-throughput sequencing will allow us to perform systematic genetic analysis of both either early stage tumors and advanced malignancies.

\section{Making sense of targeting the DNA repair machinery during the course of HIV-1 infection}

\subsection{HIV-1 integration}

As all retroviruses, HIV-1 needs to integrate its DNA into the host genome. This crucial step in the life cycle of HIV-1 is mediated by both viral and cellular proteins. It is now well described that HIV-1 subverts the DNA repair machinery to facilitate the integration of its genome [62]. Once entered into the cell the second step of the viral cycle is the reverse transcription of the viral RNA into double-stand DNA copy (cDNA). In the third integration step the cDNA is inserted into the host cell genome. The integration process takes place within a linear form of the DNA showing a Long Terminal Repeat (LTR) sequence at both ends of the host genome. The two $5^{\prime}$ and $3^{\prime}$ LTRs are recognized by the viral integrase (IN) which is part of the pre-integration complex. The process of integration is performed by the viral integrase (IN) which is introduced into the cell along other HIV-1-encoded proteins such as the reverse transcriptase and the key virulence factor Vpr. The viral IN associates to cellular factors including the chromatin binding protein lens epithelium-derived growth factor p75 (LEDGF/p75) which is essential for DNA integration. This complex associates to chromatin mainly at active transcription sites [63]. Interestingly, epigenetic marks such as $\mathrm{H} 3$ and $\mathrm{H} 4$ acetylation are more frequently seen in the integrations sites which suggest that the histone code of the chromatin is probably important for an efficient integration of the HIV-1 genome 
[64]. The integrase which contains endonuclease activity cleaves the host cell DNA and allows integration to occur [65]. In addition to IN, the key virulence factor Vpr which is introduced in the infected cell along IN, was recently shown to induce DNA damage such as DSB [66]. This may further allow the viral genome to integrate into the host cell genome. Indeed, $\mathrm{Vpr}$ is associated to several events of DNA repair i.e. phosphorylation of Histone H2AX, activation of the ATM and ATR pathways... [67]. Vpr might induce structural alteration of DNA through the degradation of host cell DNA repair proteins such as UNG2 and SLX4. A recent paper suggested that chromatin modification in resting cells such as macrophages occurred through a new mechanism involving $\mathrm{Vpr}$ [66].

Integration takes place in two distinct steps i.e. processing and joining. The integration stricto sensu is then followed by a post-integration repair which is a cellular response to DSBs. This process involves host cell DNA repair proteins such as the DNA-dependent Protein Kinase (DNA-PK) complex, ATM and ATR. All three complexes have kinase activities and they are involved in the phosphorylation of Histone $\operatorname{H2AX}[68,69]$ which is an epigenetic mark required for the recruitment of DNA repair proteins at the site of DNA damage.

\subsection{HIV-1 exploits host DNA repair proteins to complete integration}

During the first step of integration, called processing, the integrase removes two nucleotides from the $3^{\prime}$ end of the viral DNA. During the second step of integration, the joining, the integrase catalyzes a complex reaction which joins the $3^{\prime}$ end of viral DNA into the host cell DNA. Following these steps of integration, some cellular factors are subverted by the virus, which induce post-integration repair which are thought to prevent auto-integration and to enhance the efficiency of integration [68] (Fig. 3).

Two mechanisms operate in the completion of integration: HR and NHEJ. It is still debated whether the HR by LEDGF/75 or the NHEJ is involved in the completion of integration $[62,68]$. The choice between these two mechanisms of DNA repair might depend of the cell cycle status of the infected cells. HR is restricted to S and G2 phases [70] while NHEJ is thought to be efficient mainly during G1/early S phase.

Several lines of evidence point out to the importance of PIKK in the viral genome integration process of NHEJ pathway. By using NHEJdeficient fibroblasts (deficient in Ku70, Ku80, DNA-PK catalytic subunit, XRCC4 and Ligase 4), Daniel et al showed that the retroviral infection efficiency drastically decreased [71]. Moreover 80 to $90 \%$ of these cells died by apoptosis. It has been hypothesized that retroviral DNA integration is sensed by the host cell as DNA damage. Moreover, one component of the NHEJ protein complex, Ku80, when down regulated, is associated with a drastic decrease of HIV-1 infection $[72,73]$. Interestingly, this phenomenon occurs in the presence of HIV-1 IN.

Besides DNA damage sensing, the components of NHEJ are thought to support the final steps of integration. This process starts with the binding of two Ku heterodimer to the two ends of the DSB and is followed by the recruitment of the DNA-PKcs and the creation of blunt ends. Subsequently, the XRCC4/Ligase 4 is recruited close to the $\mathrm{Ku}$ heterodimer which drives ligation and allows integration to be completed. Interestingly, one member of the DNA-PK complex (Ku70) interacts with IN and prevents its proteasomal degradation [74]. Ku70 is also implicated in the activation of HIV-1 gene transcription [75]. These latter mechanisms add new insight of additional functions of Ku70 which has been also known to increase HIV-1 replication by other mechanism than retroviral DNA integration [76].

We do not have yet a clear picture of the mechanisms involved in the post-integration. For example, the importance of HR and NHEJ is still a matter of controversy and might depend of the type of host cells infected (highly replicative T CD4 + cells versus resting macrophages) and the cell environment (pro-inflammatory versus anti-inflammatory cytokines). The importance of the DNA damage sensors such as ATM

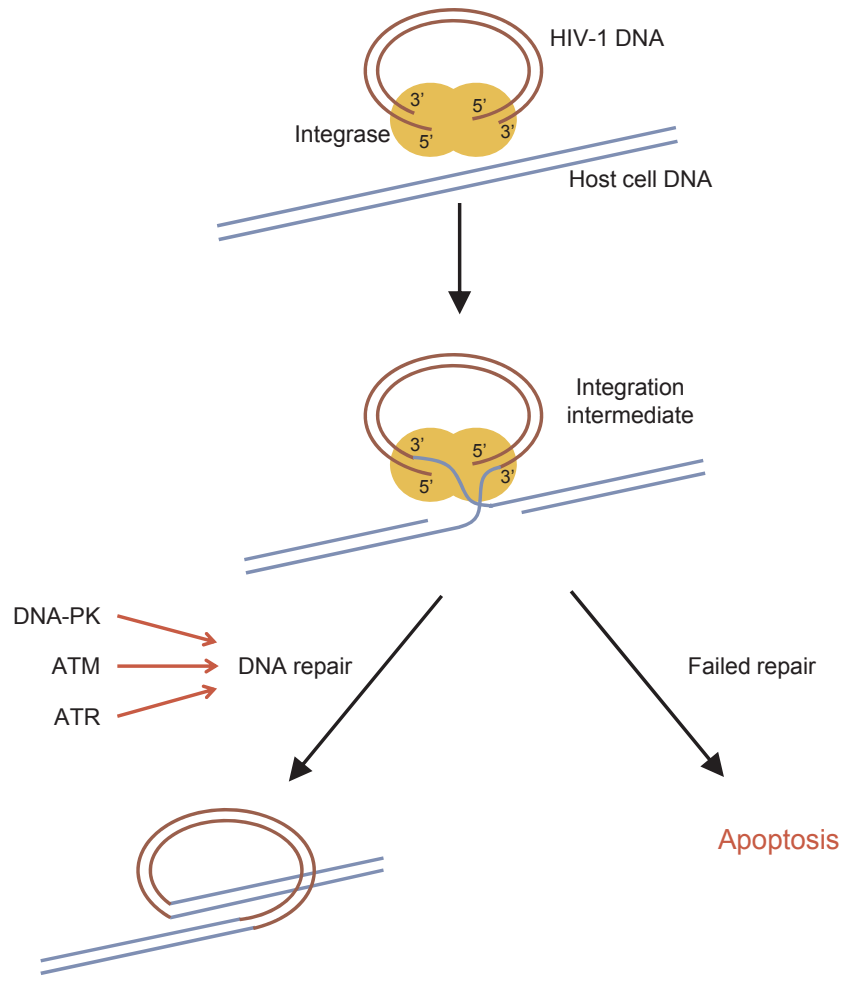

Integrated HIV-1 DNA (repaired)

Fig. 3. HIV-1 post-integration repair. The HIV-1 IN (shown in yellow) removes two nucleotides from the $3^{\prime}$ ends of the HIV-1 DNA (shown in red) and leaves $5^{\prime}$ overhangs extremities. During the joining, the integrase catalyzes a complex reaction which joins the $3^{\prime}$ end of viral DNA into the host cell DNA (shown in blue). The host cell DNA repair proteins DNA-PK, ATM and ATR, facilitate the post-integration repair and it results in a fully integrated provirus. Failure in post-integration repair results in apoptosis and cell death. (For interpretation of the references to colour in this figure legend, the reader is referred to the web version of this article.)

and ATR are as well debated however it might reflect the differences of cell models used in the different laboratories [77]. A better comprehension of the nature of the host cell DNA repair proteins involved in HIV-1 integration and post-integration processes should come from experiments designed in relevant cell models used for HIV studies, i.e. T CD4 + and macrophage cells which are the main targets of HIV-1 in infected patients (discussed in [78]).

\subsection{DNA repair and transcription}

The Ku heterodimer is involved in the regulation of HIV-1 transcription following completed integration [78]. Roles in the activation of HIV-1 transcription by the Ku proteins have been reported in a human $\mathrm{T}$ cell culture [72] and in a human colon cancer cell line (HCT116) [73].

Several other host cell DNA proteins have been shown associated with the DNA-PK complex (Fig. 4) such as the already mentioned ATM and ATR but also HDAC4, PARP1 and TOP2B [79]. Of note, it has been shown by immunoprecipitation that Ku70 is associated with TOP2B and PARP1. TOP2B is a type II DNA topoisomerase which is involved in various cell processes including DNA repair and transcription. Interestingly, HIV-1 subverts TOP2B during the course of reverse transcription and transcription of the HIV-1 life cycle [79,80]. Indeed, overexpression of TOP2B leads to the increase of viral transcription and replication [81]. One mechanism underlying this effect is the facilitation of transcription by the association of TOP2B, Ku70 and PARP1 with the viral transactivator Tat on the HIV-1 LTR promoter. TOP2B also 


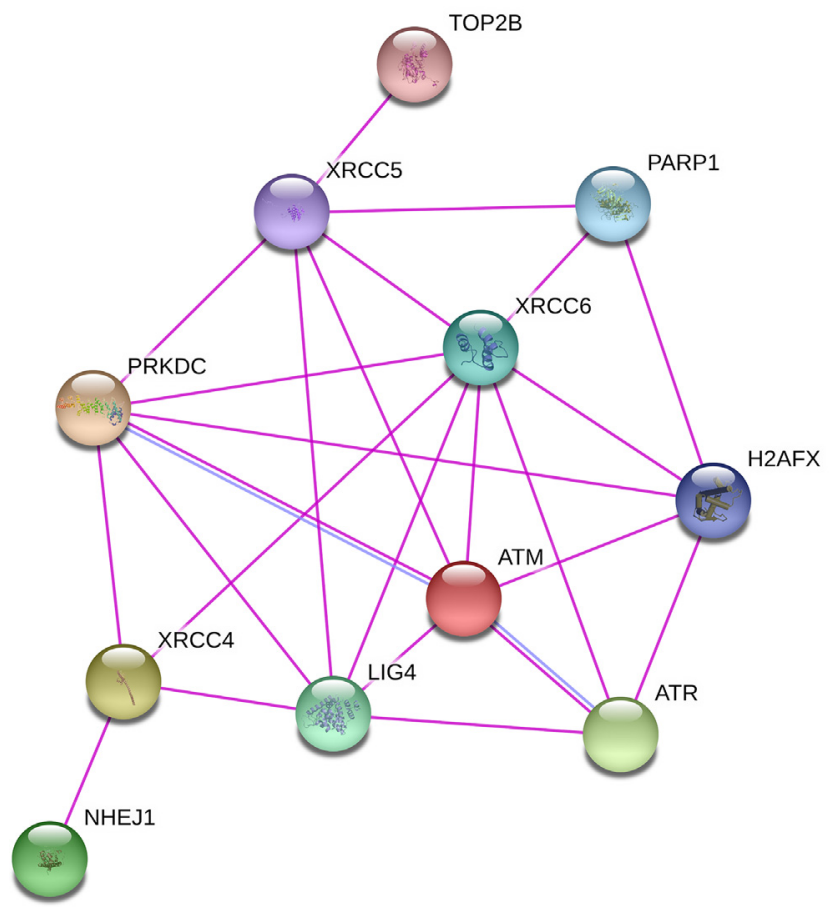

Fig. 4. DNA-PK complex protein interactions. The figure has been generated with the online software STRING (http://string-db.org). Nodes represent protein-coding gene loci (PRKDC code for DNA-PKcs, XRCC5 for Ku70, XRCC6 for Ku80, NHEJ1 for XLF, H2AFX for H2AX). Edges represent protein-protein associations. Pink edges show known interaction experimentally determined. Blue edges show known interactions for curated database. Beyond interactions between NHEJ component, DNA-PK interact with the two cell cycle regulators ATM and ATR, with the chromatin component H2AX and with TOP2B and PARP1. (For interpretation of the references to colour in this figure legend, the reader is referred to the web version of this article.)

plays a role in the regulation of the transcription of several genes by associating with DNA repair proteins such Ku70 and PARP1 [79,80]. Due to its role of topoisomerase, TOP2B might reorganize the chromatin architecture when associated to DNA damage and DNA repair proteins such as Ku70 and PARP1. It is supposed that the process of DNA repair is optimized by TOP2B which reduces DNA twisting observed during DNA damage but also during DNA replication and transcription. It is really important to note that the various steps of the HIV1 life cycle are interconnected and involve both viral and cellular proteins. TOP2B which is associated with proteins of the DNA-PK complex is a good example since it is involved in at least two steps of the HIV-1 cycle: reverse transcription and transcription. This protein constitutes therefore a promising target to combat HIV-1 [82]. There are even more connections between the DNA repair complex involved in NHEJ and transcription with the transactivator Tat entering in the game. Several papers showed that DNA-PK and the RNA pol II interact functionally and play an important role in HIV-1 transcription [83]. In addition, it has been shown that Tat also interacts with the DNA-PKcs to activate HIV-1 gene transcription [84]. Tat modulation is the result of phosphorylation on Ser16 residue by CDK2 and DNA-PK [85]. Interestingly, Tat inhibits expression of the DNA-PKcs and therefore prevents DNA repair to work efficiently. As an outcome, cells infected by HIV-1 are more sensitive to ionizing radiation [86].

\subsection{DNA-PK complex and associated proteins are involved in HIV-1 latency}

While previous work showed that Ku70/Ku80 activated HIV-1 transcription, more recent works reported that Ku exerted opposite effects on HIV-1 transcription. Indeed, Ku80 facilitates proviral silencing by inducing the formation of heterochromatin as already described for other genes in a cellular model of HIV-1 pseudo latency (human U1 cells) [87].This observation is consistent with the role exerted by PARP1 which can interact with Ku80 [88]. Indeed, PARP1 has been involved in HIV-1 transcriptional repression by inducing heterochromatin formation, the compact-repressive form of the chromatin. $\mathrm{Ku}$ proteins and PARP1 activities have been associated with repressive epigenetic marks such as histone deacetylation and DNA methylation. Therefore, these factors might be involved in the establishment of HIV1 latency [75,89]. To resolve the discrepancy on the impact of the Ku proteins on HIV-1 expression and latency needs further experiments since targeting these proteins have been shown to have opposite effects (reactivation of HIV-1 latency or repression of HIV-1 replication). In my opinion, it is important to use pertinent cell models, e.g. CD4 T cell line or cells of the monocyte-macrophage lineage to study the role of the heterodimer Ku in HIV-1 transcription. These cells are the major targets of HIV-1 and constitute therefore the main reservoirs of latently-infected cells in patient under cART [90]. Furthermore, studies of the post-translational modifications of Ku70 and Ku80 proteins showed that phosphorylation of Ku70 by cyclinB1/CDK2 disrupts Ku heterodimerization in a cell cycle dependent manner [91]. Exploring the influence of post-translational modifications of these proteins might contribute to a better comprehension of the dual role observed in the different cell models. Another factor, the histone deacetylase 4 (HDAC4) has been shown to play a role in cellular DSB by inducing the appearance of repressive marks associated with HIV-1 latency. HDAC4 is bound to the HIV-1 DNA at the site of integration [64]. Its mechanisms of action is not fully understood as it may have an indirect role as a scaffold for HDAC class I proteins or a direct role by its catalytic activity (deacetylase activity). Whatever its mechanism of action, a depletion of HDAC4 is associated to the reactivation of the latent virus in pertinent model for HIV-1 latency such as T lymphocytic cells. Intriguingly, this protein is not expressed in the promonocytic U1 cell line which argues that the mechanisms involved in the establishment and persistence of HIV-1 latency depend on the nature of the reservoirs [92,93]. An additional role for HDAC4 but also HDAC6, which is associated to the DNA repair machinery, is the protection of the cell from irradiatedinduced damages [94]. Since HDACs are involved in the establishment and persistence of HIV-1 latency in CD4 T cell line their inhibition could constitute a good strategy. Another advantage of HDAC4 inhibitors is that by targeting HDAC4 we should induce both the shock (the reactivation) and the kill (the death of the reactivated cell) when HDAC4 inhibitors are associated with radiation therapy.

\subsection{DNA damage and HIV-1 latency}

Interestingly, deficiencies in DNA repair have been described in HIV-1 latently-infected cells such as T cells [95]. These cells are more susceptible to drugs such as etoposide in inducing damages in DNA which could lead to apoptosis of cells infected with HIV-1. The mechanism of action is however not yet understood. It might involve expression of very low level of viral proteins i.e. Tat or $\mathrm{Vpr}$ as seen in the macrophage reservoirs [96]. The hypothesis of low viral replication in latent reservoirs is however debated since the discovery that genetic sequence of HIV-1 in these reservoirs does not evolve which would argue against a residual viral replication [97]. Non coding TAR microRNA which is expressed during latent infection is also evoked. This effect is related to the down regulation of ERCC which has been shown to be important in DSB repair [98].

\subsection{Therapeutic implications}

The importance of the DNA-PK complex and its associated proteins during the course of HIV-1 infection is far to be fully understood. Moreover some members of this complex such as the Ku heterodimer have dual roles in the regulation of HIV-1 transcription and replication. 
A better comprehension of its implication is therefore crucial and deserves more studies since the proteins of this complex constitute potential targets for a HIV-1 treatment. We have already mentioned the possibility to target cellular factors such as TOP2B and Ku70 which are associated to the viral transactivator Tat. Of note, Tat impairs the expression of the DNA-PK expression in productive cells which renders these infected cells more sensitive to ionizing radiation. Since NHEJ is induced during the process of integration, targeting the proteins involved in this process will even render infected cells more sensitive to ionizing radiation. This approach deserves to be explored since it is already used in preclinical and clinical evaluation of several cancer treatments [99]. We should be aware that this promising strategy might be more complicated to apply with reservoirs mainly composed of blood circulating memory T cells.

Targeting the DNA-PK complex and associated proteins might even be exploited in strategies aiming to eradicate the latently-infected reservoirs. Indeed, a deficiency in DNA repair response has already been described in latently-infected T cells [95]. As a consequence these latently-cells are more susceptible to drugs such as etoposide or NRTI which promote more damages in DNA of infected compared to un-infected cells and favor the apoptosis of the infected cells. Very interestingly, this strategy may contribute to the elimination of the HIV-1 reservoirs without prior reactivation and so deserves more attention. However, optimism has to be tempered since these effects were observed when very high concentration of drugs were used (at least 10 time more than usually assumed) which can lead to severe side effects such as cancer. Designing new drugs more efficient at lower concentration and using ionizing radiation are ways to explore since this later approach might render very vulnerable latently-infected cells to DSBs and apoptosis. This strategy could potentially lead to the elimination or to the reduction of the pool of HIV-1 reservoirs without the need of reactivation [95]. However, this strategy seems cell specific and does not work in all reservoirs, e.g. in macrophages. However, we can associate a combination of low to moderate dose of ionizing radiation with Latency Reversing Agents (LRAs) to eliminate these reservoirs. Thus, we might expect an increase of the rate of apoptosis/death of all the latently-infected reservoirs [100]. The modulation of apoptosis might increase the efficiency of the later approaches. Indeed an intriguing feature of latently-infected cells is that they are resistant to apoptosis. Latency is associated with an increase of anti-apoptotic proteins and/or to a decrease of pro-apoptotic proteins. Therefore, modulation of apoptosis could be a new way to target latently-infected cells and constitute an original therapeutic approach to eliminate infected cells [101].

Radiation-induced DNA damage, which induces the NHEJ machinery, might allow us to specifically target infected cells especially the latently-infected ones. This is important since we do not have specific treatment that targets the latently-infected cells. Indeed, apart from CD32a, a low affinity receptor for immunoglobulin G Fc fragment recently described as a specific biomarker for CD4 + T cell HIV-reservoir [102], there is a crucial lack of specific biomarkers for latently infected cells. Indeed, even the CD32a marker is a matter of controversy with several papers challenging with their finding $[103,104]$. However, a recent communication at the 2018 Frontier of Retrovirology congress corroborated the results of the group of Benkirane by showing with another approach that indeed $\mathrm{CD} 32+\mathrm{CD} 4+\mathrm{T}$ cells are enriched in HIV-1 DNA [105]. They notably stress the importance of getting purer CD32 + CD4 + cells fraction for analysis. The lack in the optimization of the assays which aim to isolate purer fractions of CD32+ D4 + might explain the absence of enrichment in HIV-1 DNA in these later cells. Combination of strategies should improve treatment outcome as well. We should consider a combination of strategies such as (1) increasing the vulnerability of the latently-infected cells by targeting the DNA-PK complex and associated proteins involved in NHEJ jointly with (2) strategies targeting proteins involved in apoptosis or (3) reactivating latently-infected cells by LRAs since ionizing radiation alone cannot reactivate the dormant virus in all the reservoirs. All in all, targeting the cellular components of the DNA-PK and associated proteins make sense to combat HIV-1 in both productive infected and latently-infected cells. Moreover, this strategy has the main benefit to circumvent drug-resistance problems that arise with drugs raised against HIV-1 proteins.

\section{Competing interests}

None.

\section{Acknowledgements}

We are grateful to Andrea Janossy for careful and critical reading of the manuscript. This work in OR and CS laboratory was supported by grants from the French agency for research on AIDS and viral hepatitis (ARNS), Sidaction, the European Union's Horizon 2020 research and innovation program under grant agreement No 691119 EU4HIVCURE - H2020-MSCA-RISE-2015, Alsace contre le Cancer and Institut Universitaire de France.

\section{References:}

[1] T. Lindahl, Instability and decay of the primary structure of DNA, Nature 362 (1993) 709-715, https://doi.org/10.1038/362709a0.

[2] D.G. Schatz, P.C. Swanson, V(D)J recombination: mechanisms of initiation, Annu. Rev. Genet. 45 (2011) 167-202, https://doi.org/10.1146/annurev-genet-110410132552.

[3] L. Nicolas, M. Cols, J.E. Choi, J. Chaudhuri, B. Vuong, P. Casali, N.L. Cols, C. Je, Open Peer Review Generating and repairing genetically programmed DNA breaks during immunoglobulin class switch recombination [version 1; referees: 2 approved], F1000 Fac Rev. 7 (2018) 458458-458513, https://doi.org/10.12688/ f1000research.13247.1.

[4] G. Giglia-Mari, A. Zotter, W. Vermeulen, DNA damage response, Cold Spring Harb. Perspect. Biol. 3 (2011) a000745, , https://doi.org/10.1101/cshperspect. a000745.

[5] J.R. Chapman, M.R.G. Taylor, S.J. Boulton, Playing the end game: DNA doublestrand break repair pathway choice, Mol. Cell. 47 (2012) 497-510, https://doi. org $/ 10.1016 /$ j.molcel.2012.07.029.

[6] Z. Mao, M. Bozzella, A. Seluanov, V. Gorbunova, Comparison of nonhomologous end joining and homologous recombination in human cells, DNA Repair (Amst.) 7 (2008) 1765-1771, https://doi.org/10.1016/j.dnarep.2008.06.018.

[7] S. Lees-Miller, Repair of DNA double strand breaks by non-homologous end joining, Biochimie 85 (2003) 1161-1173, https://doi.org/10.1016/j.biochi.2003. 10.011 .

[8] J.-H. Seol, E.Y. Shim, S.E. Lee, Microhomology-mediated end joining: Good, bad and ugly, Mutat. Res. 809 (2018) 81-87, https://doi.org/10.1016/j.mrfmmm. 2017.07.002.

[9] J.R. Walker, R.A. Corpina, J. Goldberg, Structure of the Ku heterodimer bound to DNA and its implications for double-strand break repair, Nature 412 (2001) 607-614, https://doi.org/10.1038/35088000.

[10] P.-O. Mari, B.I. Florea, S.P. Persengiev, N.S. Verkaik, H.T. Brüggenwirth, M. Modesti, G. Giglia-Mari, K. Bezstarosti, J.A.A. Demmers, T.M. Luider, A.B. Houtsmuller, D.C. van Gent, Dynamic assembly of end-joining complexes requires interaction between Ku70/80 and XRCC4, Proc. Natl. Acad. Sci. U.S.A. 103 (2006) 18597-18602, https://doi.org/10.1073/pnas.0609061103.

[11] J.F. Goodwin, K.E. Knudsen, Beyond DNA repair: DNA-PK function in cancer, Cancer Discov. 4 (2014) 1126-1139, https://doi.org/10.1158/2159-8290.CD-140358.

[12] B.L.L. Mahaney, K. Meek, S.P.P. Lees-Miller, Repair of ionizing radiation-induced DNA double-strand breaks by non-homologous end-joining, Biochem. J. 417 (2009) 639-650, https://doi.org/10.1042/BJ20080413.

[13] A.J. Davis, B.P.C. Chen, D.J. Chen, DNA-PK: a dynamic enzyme in a versatile DSB repair pathway, DNA Repair (Amst.) 17 (2014) 21-29, https://doi.org/10.1016/j. dnarep.2014.02.020.

[14] N. Jette, S.P. Lees-Miller, The DNA-dependent protein kinase: a multifunctional protein kinase with roles in DNA double strand break repair and mitosis, Prog. Biophys. Mol. Biol. 117 (2015) 194-205, https://doi.org/10.1016/j.pbiomolbio. 2014.12.003.

[15] Q. Ding, Y.V.R. Reddy, W. Wang, T. Woods, P. Douglas, D.A. Ramsden, S.P. Lees Miller, K. Meek, Autophosphorylation of the catalytic subunit of the DNA-dependent protein kinase is required for efficient end processing during DNA doublestrand break repair, Mol. Cell. Biol. 23 (2003) 5836-5848, https://doi.org/10. 1128/MCB.23.16.5836-5848.2003.

[16] V.L. Fell, C. Schild-Poulter, The Ku heterodimer: function in DNA repair and beyond, Mutat. Res. 763 (2015) 15-29, https://doi.org/10.1016/j.mrrev.2014.06. 002 .

[17] G.J. Grundy, H.A. Moulding, K.W. Caldecott, S.L. Rulten, One ring to bring them all-the role of $\mathrm{Ku}$ in mammalian non-homologous end joining, DNA Repair (Amst.) 17 (2014) 30-38, https://doi.org/10.1016/j.dnarep.2014.02.019. 
[18] L.J. Kuo, L.-X. Yang, Gamma-H2AX - a novel biomarker for DNA double-strand breaks, In Vivo 22 (n.d.) 305-309. http://www.ncbi.nlm.nih.gov/pubmed/ 18610740 (accessed August 31, 2018)

[19] M.W. Luczak, A. Zhitkovich, Monoubiquitinated $\gamma$-H2AX: abundant product and specific biomarker for non-apoptotic DNA double-strand breaks, Toxicol. Appl. Pharmacol. 355 (2018), https://doi.org/10.1016/j.taap.2018.07.007.

[20] A. Georgoulis, C.E. Vorgias, G.P. Chrousos, E.P. Rogakou, Genome instability and $\gamma$ H2AX, Int. J. Mol. Sci. 18 (2017) 1-10, https://doi.org/10.3390/ijms18091979.

[21] R. Ceccaldi, B. Rondinelli, A.D. D'Andrea, Repair pathway choices and consequences at the double-strand break, Trends Cell Biol. 26 (2015) 52-64, https:// doi.org/10.1016/j.tcb.2015.07.009.

[22] K.K. Khanna, S.P. Jackson, DNA double-strand breaks: signaling, repair and the cancer connection, Nat. Genet. 27 (2001) 247-254, https://doi.org/10.1038/ 85798.

[23] M.J. O'Connor, Targeting the DNA damage response in cancer, Mol. Cell. 60 (2015) 547-560, https://doi.org/10.1016/j.molcel.2015.10.040.

[24] V. Marcel, F. Nguyen Van Long, J.-J. Diaz, 40 Years of research put p53 in translation, Cancers (Basel) 10 (2018) doi:10.3390/cancers10050152.

[25] J. Saha, A.J. Davis, Unsolved mystery: the role of BRCA1 in DNA end-joining, J Radiat. Res. 57 (Suppl. 1) (2016) i18-i24, https://doi.org/10.1093/jrr/rrw032.

[26] T.D. Halazonetis, V.G. Gorgoulis, J. Bartek, An oncogene-induced DNA damage model for cancer development, Science (80-) 319 (2008) 1352-1355, https://doi. org $/ 10.1126 /$ science 1140735 .

[27] J. Bartkova, Z. Horejsí, K. Koed, A. Krämer, F. Tort, K. Zieger, P. Guldberg, M. Sehested, J.M. Nesland, C. Lukas, T. Ørntoft, J. Lukas, J. Bartek, DNA damage response as a candidate anti-cancer barrier in early human tumorigenesis, Nature 434 (2005) 864-870, https://doi.org/10.1038/nature03482.

[28] P.J. Stephens, D.J. McBride, M.-L. Lin, I. Varela, E.D. Pleasance, J.T. Simpson, L.A. Stebbings, C. Leroy, S. Edkins, L.J. Mudie, C.D. Greenman, M. Jia, C. Latimer, J.W. Teague, K.W. Lau, J. Burton, M.A. Quail, H. Swerdlow, C. Churcher, R. Natrajan, A.M. Sieuwerts, J.W.M. Martens, D.P. Silver, A. Langerød, H.E.G. Russnes, J.A. Foekens, J.S. Reis-Filho, L. van 't Veer, A.L. Richardson, A.L. Børresen-Dale, P.J. Campbell, P.A. Futreal, M.R. Stratton, Complex landscapes of somatic rearrangement in human breast cancer genomes, Nature 462 (2009) 1005-1010, https://doi.org/10.1038/nature08645.

[29] N.J. Curtin, DNA repair dysregulation from cancer driver to therapeutic target, Nat. Rev. Cancer 12 (2012) 801-817, https://doi.org/10.1038/nrc3399.

[30] M. Shaheen, C. Allen, J.A. Nickoloff, R. Hromas, Synthetic lethality: exploiting the addiction of cancer to DNA repair, Blood 117 (2011) 6074-6082, https://doi.org/ 10.1182/blood-2011-01-313734.

[31] B.J. Sishc, A.J. Davis, The role of the core non-homologous end joining factors in carcinogenesis and cancer, Cancers (Basel) 9 (2017) 81, https://doi.org/10.3390/ cancers 9070081.

[32] D.H. Auckley, R.E. Crowell, E.R. Heaphy, C.A. Stidley, J.F. Lechner, F.D. Gilliland, S.A. Belinsky, Reduced DNA-dependent protein kinase activity is associated with lung cancer, Carcinogenesis 22 (2001) 723-727 (accessed August 31, 2018).

[33] P. Mazzarelli, P. Parrella, D. Seripa, E. Signori, G. Perrone, C. Rabitti, D. Borzomati, A. Gabbrielli, M.G. Matera, C. Gravina, M. Caricato, M.L. Poeta, M. Rinaldi, S. Valeri, R. Coppola, V.M. Fazio, DNA end binding activity and Ku70/ 80 heterodimer expression in human colorectal tumor, World J. Gastroenterol. 11 (2005) 6694-6700, https://doi.org/10.3748/WJG.V11.I42.6694.

[34] H. Hu, Y. Zhang, M. Zou, S. Yang, X.-Q. Liang, Expression of TRF1, TRF2, TIN2, TERT, KU70, and BRCA1 proteins is associated with telomere shortening and may contribute to multistage carcinogenesis of gastric cancer, J. Cancer Res. Clin. Oncol. 136 (2010) 1407-1414, https://doi.org/10.1007/s00432-010-0795-x.

[35] W.M. Bonner, C.E. Redon, J.S. Dickey, A.J. Nakamura, O.A. Sedelnikova, S. Solier, Y. Pommier, GammaH2AX and cancer, Nat. Rev. Cancer. 8 (2008) 957-967, https://doi.org/10.1038/nrc2523.

[36] C. Gullo, M. Au, G. Feng, G. Teoh, The biology of Ku and its potential oncogenic role in cancer, Biochim. Biophys. Acta 1765 (2006) 223-234, https://doi.org/10. 1016/j.bbcan.2006.01.001.

[37] M. Pospisilova, M. Seifrtova, M. Rezacova, Small molecule inhibitors of DNA-PK for tumor sensitization to anticancer therapy, J. Physiol. Pharmacol. 68 (2017) 337-344.

[38] T. Tsuji, L.M. Sapinoso, T. Tran, B. Gaffney, L. Wong, S. Sankar, H.K. Raymon, D.S. Mortensen, S. Xu, CC-115, a dual inhibitor of mTOR Kinase and DNA-PK, blocks DNA damage repair pathways and selectively inhibits ATM-deficient cell growth in vitro, Oncotarget 8 (2017) 74688-74702, https://doi.org/10.18632/ oncotarget.20342.

[39] J. Biau, F. Devun, W. Jdey, E. Kotula, M. Quanz, E. Chautard, M. Sayarath, J.S. Sun, P. Verrelle, M. Dutreix, A preclinical study combining the DNA repair inhibitor Dbait with radiotherapy for the treatment of melanoma, Neoplasia 16 (2014) 835-844, https://doi.org/10.1016/j.neo.2014.08.008.

[40] C. Le Tourneau, B. Dreno, Y. Kirova, J.J. Grob, T. Jouary, C. Dutriaux, L. Thomas, C. Lebbé, L. Mortier, P. Saiag, M.F. Avril, E. Maubec, P. Joly, P. Bey, J.M. Cosset, J.S. Sun, B. Asselain, F. Devun, M.E. Marty, M. Dutreix, First-in-human phase I study of the DNA-repair inhibitor DT01 in combination with radiotherapy in patients with skin metastases from melanoma, Br. J. Cancer 114 (2016) 1199-1205, https://doi.org/10.1038/bjc.2016.120.

[41] N.I. Herath, F. Devun, M.-C. Lienafa, A. Herbette, A. Denys, J.-S. Sun, M. Dutreix, The DNA repair inhibitor DT01 as a novel therapeutic strategy for chemosensitization of colorectal liver metastasis, Mol. Cancer Ther. 15 (2016) 15-22, https:// doi.org/10.1158/1535-7163.MCT-15-0408.

[42] S. Thierry, W. Jdey, S. Alculumbre, V. Soumelis, P. Noguiez-Hellin, M. Dutreix, The DNA repair inhibitor Dbait is specific for malignant hematologic cells in blood, Mol. Cancer Ther. 16 (2017) 2817-2827, https://doi.org/10.1158/1535-
7163.MCT-17-0405.

[43] M. Mognato, L. Celotti, MicroRNAs used in combination with anti-cancer treatments can enhance therapy efficacy, Mini Rev. Med. Chem. 15 (2015) 1052-1062, https://doi.org/10.2174/1389557515666150709115355.

[44] D. Yan, W.L. Ng, X. Zhang, P. Wang, Z. Zhang, Y.-Y. Mo, H. Mao, C. Hao, J.J. Olson, W.J. Curran, Y. Wang, Targeting DNA-PKcs and ATM with miR-101 sensitizes tumors to radiation, PLoS One 5 (2010) e11397, , https://doi.org/10. 1371/journal.pone.0011397.

[45] H. Hu, Y. He, Y. Wang, W. Chen, B. Hu, Y. Gu, micorRNA-101 silences DNA-PKcs and sensitizes pancreatic cancer cells to gemcitabine, Biochem. Biophys. Res. Commun. 483 (2017) 725-731, https://doi.org/10.1016/j.bbrc.2016.12.074.

[46] N. Li, Y. Ma, L. Ma, Y. Guan, L. Ma, D. Yang, MicroRNA-488-3p sensitizes malignant melanoma cells to cisplatin by targeting PRKDC, Cell Biol. Int. 41 (2017) 622-629, https://doi.org/10.1002/cbin.10765.

[47] E. Weterings, A.C. Gallegos, L.N. Dominick, L.S. Cooke, T.N. Bartels, J. Vagner, T.O. Matsunaga, D. Mahadevan, A novel small molecule inhibitor of the DNA repair protein Ku70/80, DNA Repair (Amst.) 43 (2016) 98-106, https://doi.org/ 10.1016/j.dnarep.2016.03.014.

[48] I.S. Ayene, L.P. Ford, C.J. Koch, Ku protein targeting by Ku70 small interfering RNA enhances human cancer cell response to topoisomerase II inhibitor and gamma radiation, Mol. Cancer Ther. 4 (2005) 529-536, https://doi.org/10.1158/ 1535-7163.MCT-04-0130.

[49] P.C. Fong, T.A. Yap, D.S. Boss, C.P. Carden, M. Mergui-Roelvink, C. Gourley, J. De Greve, J. Lubinski, S. Shanley, C. Messiou, R. A'Hern, A. Tutt, A. Ashworth, J. Stone, J. Carmichael, J.H.M. Schellens, J.S. de Bono, S.B. Kaye, Poly(ADP)-ribose polymerase inhibition: frequent durable responses in BRCA carrier ovarian cancer correlating with platinum-free interval, J. Clin. Oncol. 28 (2010) 2512-2519, https://doi.org/10.1200/JCO.2009.26.9589.

[50] H. Farmer, N. McCabe, C.J. Lord, A.N.J. Tutt, D.A. Johnson, T.B. Richardson, M. Santarosa, K.J. Dillon, I. Hickson, C. Knights, N.M.B. Martin, S.P. Jackson, G.C.M. Smith, A. Ashworth, Targeting the DNA repair defect in BRCA mutant cells as a therapeutic strategy, Nature 434 (2005) 917-921, https://doi.org/10.1038/ nature03445.

[51] W. Sakai, E.M. Swisher, B.Y. Karlan, M.K. Agarwal, J. Higgins, C. Friedman, E. Villegas, C. Jacquemont, D.J. Farrugia, F.J. Couch, N. Urban, T. Taniguchi, Secondary mutations as a mechanism of cisplatin resistance in BRCA2-mutated cancers, Nature 451 (2008) 1116-1120, https://doi.org/10.1038/nature06633.

[52] A.G. Patel, J.N. Sarkaria, S.H. Kaufmann, Nonhomologous end joining drives poly (ADP-ribose) polymerase (PARP) inhibitor lethality in homologous recombinationdeficient cells, Proc. Natl. Acad. Sci. U.S.A. 108 (2011) 3406-3411, https://doi. org /10.1073/pnas.1013715108.

[53] P. Bouwman, A. Aly, J.M. Escandell, M. Pieterse, J. Bartkova, H. van der Gulden, S. Hiddingh, M. Thanasoula, A. Kulkarni, Q. Yang, B.G. Haffty, J. Tommiska, C. Blomqvist, R. Drapkin, D.J. Adams, H. Nevanlinna, J. Bartek, M. Tarsounas, S. Ganesan, J. Jonkers, 53BP1 loss rescues BRCA1 deficiency and is associated with triple-negative and BRCA-mutated breast cancers, Nat. Struct. Mol. Biol. 17 (2010) 688-695, https://doi.org/10.1038/nsmb.1831.

[54] N. Albarakati, T.M.A.A. Abdel-Fatah, R. Doherty, R. Russell, D. Agarwal, P. Moseley, C. Perry, A. Arora, N. Alsubhi, C. Seedhouse, E.A. Rakha, A. Green, G. Ball, S. Chan, C. Caldas, I.O. Ellis, S. Madhusudan, Targeting BRCA1-BER deficient breast cancer by ATM or DNA-PKcs blockade either alone or in combination with cisplatin for personalized therapy, Mol. Oncol. 9 (2015) 204-217, https:// doi.org/10.1016/j.molonc.2014.08.001.

[55] F. Dietlein, L. Thelen, M. Jokic, R.D. Jachimowicz, L. Ivan, G. Knittel, U. Leeser, J. van Oers, W. Edelmann, L.C. Heukamp, H.C. Reinhardt, A functional cancer genomics screen identifies a druggable synthetic lethal interaction between MSH3 and PRKDC, Cancer Discov. 4 (2014) 592-605, https://doi.org/10.1158/21598290.CD-13-0907.

[56] H. Jiang, H.C. Reinhardt, J. Bartkova, J. Tommiska, C. Blomqvist, H. Nevanlinna, J. Bartek, M.B. Yaffe, M.T. Hemann, The combined status of ATM and p53 link tumor development with therapeutic response, Genes Dev. 23 (2009) 1895-1909, https://doi.org/10.1101/gad.1815309.

[57] M. Macheret, T.D. Halazonetis, DNA replication stress as a hallmark of cancer, Annu. Rev. Pathol. 10 (2015) 425-448, https://doi.org/10.1146/annurev-pathol012414-040424.

[58] R. Buisson, J.L. Boisvert, C.H. Benes, L. Zou, Distinct but concerted roles of ATR DNA-PK, and Chk1 in countering replication stress during S phase, Mol. Cell 59 (2015) 1011-1024, https://doi.org/10.1016/j.molcel.2015.07.029.

[59] C.J. Ryan, I. Bajrami, C.J. Lord, Synthetic lethality and cancer - penetrance as the major barrier, Trends Cancer 4 (2018) 671-683, https://doi.org/10.1016/j.trecan. 2018.08.003.

[60] N.J. O'Neil, M.L. Bailey, P. Hieter, Synthetic lethality and cancer, Nat. Rev. Genet 18 (2017) 613-623, https://doi.org/10.1038/nrg.2017.47.

[61] C. Muller, G. Christodoulopoulos, B. Salles, L. Panasci, DNA-Dependent protein kinase activity correlates with clinical and in vitro sensitivity of chronic lymphocytic leukemia lymphocytes to nitrogen mustards, Blood 92 (1998) 2213-2219 (accessed October 16, 2018).

[62] R. Craigie, F.D. Bushman, HIV DNA integration, a006890-a006890, Cold Spring Harb. Perspect. Med. 2 (2012), https://doi.org/10.1101/cshperspect.a006890.

[63] S.D. Rezaei, P.U. Cameron, Human immunodeficiency virus (HIV)-1 integration sites in viral latency, Curr. HIV/AIDS Rep. 12 (2015) 88-96, https://doi.org/10. 1007/s11904-014-0241-9.

[64] R. Daniel, J.A. Smith, Integration site selection by retroviral vectors: molecula mechanism and clinical consequences, Hum. Gene Ther. 19 (2008) 557-568, https://doi.org/10.1089/hum.2007.148.

[65] P. Hindmarsh, J. Leis, Retroviral DNA integration, Microbiol. Mol. Biol. Rev. 63 
(1999) 836-843 (table of contents).

[66] K. Iijima, J. Kobayashi, Y. Ishizaka, Structural alteration of DNA induced by viral protein R of HIV-1 triggers the DNA damage response, Retrovirology 15 (2018) 8, https://doi.org/10.1186/s12977-018-0391-8.

[67] C. Nakai-Murakami, M. Shimura, M. Kinomoto, Y. Takizawa, K. Tokunaga, T. Taguchi, S. Hoshino, K. Miyagawa, T. Sata, H. Kurumizaka, A. Yuo, Y. Ishizaka, HIV-1 Vpr induces ATM-dependent cellular signal with enhanced homologous recombination, Oncogene 26 (2007) 477-486, https://doi.org/10.1038/sj.onc 1209831.

[68] J.A. Smith, R. Daniel, Following the path of the virus: the exploitation of host DNA repair mechanisms by retroviruses, ACS Chem. Biol. 1 (2006) 217-226, https:// doi.org/10.1021/cb600131q.

[69] A. Cooper, M. García, C. Petrovas, T. Yamamoto, R.A. Koup, G.J. Nabel, HIV-1 causes CD4 cell death through DNA-dependent protein kinase during viral integration, Nature 498 (2013) 376-379, https://doi.org/10.1038/nature12274.

[70] S.P. Jackson, J. Bartek, The DNA-damage response in human biology and disease, Nature 461 (2009) 1071-1078, https://doi.org/10.1038/nature08467.

[71] R. Daniel, R.A. Katz, A.M. Skalka, A role for DNA-PK in retroviral DNA integration, Science 284 (1999) 644-647.

[72] S. Waninger, K. Kuhen, X. Hu, J.E. Chatterton, F. Wong-Staal, H. Tang, Identification of cellular cofactors for human immunodeficiency virus replication via a ribozyme-based genomics approach, J. Virol. 78 (2004) 12829-12837, https://doi.org/10.1128/JVI.78.23.12829-12837.2004.

[73] L. Jeanson, F. Subra, S. Vaganay, M. Hervy, E. Marangoni, J. Bourhis, J.F. Mouscadet, Effect of Ku80 depletion on the preintegrative steps of HIV-1 replication in human cells, Virology 300 (2002) 100-108, https://doi.org/10.1006/ viro.2002.1515.

[74] Y. Zheng, Z. Ao, B. Wang, K.D. Jayappa, X. Yao, Host protein Ku70 binds and protects HIV-1 integrase from proteasomal degradation and is required for HIV replication, J. Biol. Chem. 286 (2011) 17722-17735, https://doi.org/10.1074/ jbc.M110.184739.

[75] G. Manic, A. Maurin-Marlin, F. Laurent, I. Vitale, S. Thierry, O. Delelis, P. Dessen, M. Vincendeau, C. Leib-Mösch, U. Hazan, J.-F. Mouscadet, S. Bury-Moné, Impact of the Ku complex on HIV-1 expression and latency, PLoS One 8 (2013) e69691, , https://doi.org/10.1371/journal.pone.0069691.

[76] A.N. Anisenko, E.S. Knyazhanskaya, A.O. Zalevsky, J.Y. Agapkina, A.I. Sizov, T.S. Zatsepin, M.B. Gottikh, Characterization of HIV-1 integrase interaction with human Ku70 protein and initial implications for drug targeting, Sci. Rep. 7 (2017) 5649, https://doi.org/10.1038/s41598-017-05659-5.

[77] Y. Ariumi, P. Turelli, M. Masutani, D. Trono, DNA Damage Sensors ATM, ATR, DNA-PKcs, and PARP-1 are dispensable for human immunodeficiency virus type 1 integration, J. Virol. 79 (2005) 2973-2978, https://doi.org/10.1128/JVI.79.5 2973-2978.2005.

[78] O.A. Shadrina, E.S. Knyazhanskaya, S.P. Korolev, M. B. Gottikh, Host proteins Ku and HMGA1 as participants of HIV-1 transcription, Acta Naturae 8 (2016) 34-47 (accessed February 12, 2018).

[79] R. Mandraju, A. Chekuri, C. Bhaskar, K. Duning, J. Kremerskothen, A.K. Kondapi, Topoisomerase II $\beta$ associates with Ku70 and PARP-1 during double strand break repair of DNA in neurons, Arch. Biochem. Biophys. 516 (2011) 128-137, https:// doi.org/10.1016/j.abb.2011.10.001.

[80] B.-G. Ju, V.V. Lunyak, V. Perissi, I. Garcia-Bassets, D.W. Rose, C.K. Glass, M.G. Rosenfeld, A topoisomerase II-mediated dsDNA break required for regulated transcription, Science (80-.) 312 (2006) 1798-1802, https://doi.org/10.1126/ science.1127196.

[81] A. Chekuri, C. Bhaskar, V.S. Bollimpelli, A.K. Kondapi, TopoisomeraseII $\beta$ in HIV-1 transactivation, Arch. Biochem. Biophys. 593 (2016) 90-97, https://doi.org/10. 1016/j.abb.2016.02.009.

[82] V.S. Bollimpelli, P.S. Dholaniya, A.K. Kondapi, Topoisomerase II $\beta$ and its role in different biological contexts, Arch. Biochem. Biophys. 633 (2017) 78-84, https:// doi.org/10.1016/j.abb.2017.06.021.

[83] S. Tyagi, A. Ochem, M. Tyagi, DNA-dependent protein kinase interacts functionally with the RNA polymerase II complex recruited at the human immunodeficiency virus (HIV) long terminal repeat and plays an important role in HIV gene expression, J. Gen. Virol. 92 (2011) 1710-1720, https://doi.org/10. 1099/vir.0.029587-0.

[84] A. Ivanov, X. Lin, T. Ammosova, A.V. Ilatovskiy, N. Kumari, H. Lassiter, N. Afangbedji, X. Niu, M.G. Petukhov, S. Nekhai, HIV-1 Tat phosphorylation on Ser-16 residue modulates HIV-1 transcription, Retrovirology 15 (2018) 39, https://doi.org/10.1186/s12977-018-0422-5.

[85] S.-M. Zhang, H. Zhang, T.-Y. Yang, T.-Y. Ying, P.-X. Yang, X.-D. Liu, S.-J. Tang, P.K. Zhou, Interaction between HIV-1 Tat and DNA-PKcs modulates HIV transcription and class switch recombination, Int. J. Biol. Sci. 10 (2014) 1138-1149, https://doi.org/10.7150/ijbs.10366.

[86] Y. Sun, Y.-C. Huang, Q.-Z. Xu, H.-P. Wang, B. Bai, J.-L. Sui, P.-K. Zhou, HIV-1 Tat depresses DNA-PKCS expression and DNA repair, and sensitizes cells to ionizing radiation, Int. J. Radiat. Oncol. 65 (2006) 842-850, https://doi.org/10.1016/j. ijrobp.2006.02.040.

[87] L. Jeanson, J.-F. Mouscadet, Ku Represses the HIV-1 transcription, J. Biol. Chem. 277 (2002) 4918-4924, https://doi.org/10.1074/jbc.M110830200.

[88] S. Galande, T. Kohwi-Shigematsu, Poly(ADP-ribose) polymerase and Ku autoantigen form a complex and synergistically bind to matrix attachment sequences, J. Biol. Chem. 274 (1999) 20521-20528.

[89] M.T.D. Bueno, D. Reyes, L. Valdes, A. Saheba, E. Urias, C. Mendoza, O.I. Fregoso, M. Llano, Poly(ADP-ribose) polymerase 1 promotes transcriptional repression of integrated retroviruses, J. Virol. 87 (2013) 2496-2507, https://doi.org/10.1128/ JVI.01668-12.

[90] C. Schwartz, S. Bouchat, C. Marban, V. Gautier, C. Van Lint, O. Rohr, V. Le Douce, On the way to find a cure: Purging latent HIV-1 reservoirs, Biochem. Pharmacol. (2017), https://doi.org/10.1016/j.bcp.2017.07.001.

[91] S. Mukherjee, P. Chakraborty, P. Saha, Phosphorylation of Ku70 subunit by cell cycle kinases modulates the replication related function of Ku heterodimer, Nucleic Acids Res. 44 (2016) 7755-7765, https://doi.org/10.1093/nar/gkw622.

[92] I. Palmisano, G. Della Chiara, R.L. D’Ambrosio, C. Huichalaf, P. Brambilla, S. Corbetta, M. Riba, R. Piccirillo, S. Valente, G. Casari, A. Mai, F.M. Boneschi, D. Gabellini, G. Poli, M.V. Schiaffino, Amino acid starvation induces reactivation of silenced transgenes and latent HIV-1 provirus via down-regulation of histone deacetylase 4 (HDAC4), Proc. Natl. Acad. Sci. 109 (2012) E2284-E2293, https:// doi.org/10.1073/pnas.1202174109.

[93] I. Palmisano, G. Della Chiara, M.V. Schiaffino, G. Poli, Passport control for foreign integrated DNAs, Mob. Genet. Elements 2 (2012) 233-238, https://doi.org/10. 4161/mge.22610.

[94] F. Marampon, F. Megiorni, S. Camero, C. Crescioli, H.P. McDowell, R. Sferra, A. Vetuschi, S. Pompili, L. Ventura, F. De Felice, V. Tombolini, C. Dominici, R. Maggio, C. Festuccia, G.L. Gravina, HDAC4 and HDAC6 sustain DNA double strand break repair and stem-like phenotype by promoting radioresistance in glioblastoma cells, Cancer Lett. 397 (2017) 1-11, https://doi.org/10.1016/j. canlet.2017.03.028.

[95] D. Piekna-Przybylska, G. Sharma, S.B. Maggirwar, R.A. Bambara, Deficiency in DNA damage response, a new characteristic of cells infected with latent HIV-1, Cell Cycle 16 (2017) 968-978, https://doi.org/10.1080/15384101.2017. 1312225 .

[96] J. Brodin, F. Zanini, L. Thebo, C. Lanz, G. Bratt, R.A. Neher, J. Albert, Establishment and stability of the latent HIV-1 DNA reservoir, Elife 5 (2016), https://doi.org/10.7554/eLife. 18889.

[97] A. Kumar, W. Abbas, G. Herbein, HIV-1 latency in monocytes/macrophages, Viruses 6 (2014) 1837-1860, https://doi.org/10.3390/v6041837.

[98] Z. Klase, R. Winograd, J. Davis, L. Carpio, R. Hildreth, M. Heydarian, S. Fu, T. McCaffrey, E. Meiri, M. Ayash-Rashkovsky, S. Gilad, Z. Bentwich, F. Kashanchi, HIV-1 TAR miRNA protects against apoptosis by altering cellular gene expression, Retrovirology 6 (2009) 18, https://doi.org/10.1186/1742-4690-6-18.

[99] N.J. Curtin, Inhibiting the DNA damage response as a therapeutic manoeuvre in cancer, Br. J. Pharmacol. 169 (2013) 1745-1765, https://doi.org/10.1111/bph. 12244.

[100] S. Iordanskiy, F. Kashanchi, Potential of radiation-induced cellular stress for reactivation of latent HIV-1 and killing of infected cells, AIDS Res. Hum. Retroviruses 32 (2016) 120-124, https://doi.org/10.1089/AID.2016.0006.

[101] Y. Kim, J.L. Anderson, S.R. Lewin, Getting the "Kill" into "Shock and Kill": strategies to eliminate latent HIV, Cell Host Microbe 23 (2018) 14-26, https://doi. org/10.1016/j.chom.2017.12.004.

[102] B. Descours, G. Petitjean, J.-L. López-Zaragoza, T. Bruel, R. Raffel, C. Psomas, J. Reynes, C. Lacabaratz, Y. Levy, O. Schwartz, J.D. Lelievre, M. Benkirane, CD32a is a marker of a CD4 T-cell HIV reservoir harbouring replication-competent proviruses, Nature (2017), https://doi.org/10.1038/nature21710.

[103] M. Abdel-Mohsen, L. Kuri-Cervantes, J. Grau-Exposito, A.M. Spivak, R.A. Nell C. Tomescu, S.K. Vadrevu, L.B. Giron, C. Serra-Peinado, M. Genescà, J. Castellví, G. Wu, P.M. Del Rio Estrada, M. González-Navarro, K. Lynn, C.T. King, S. Vemula, K. Cox, Y. Wan, Q. Li, K. Mounzer, J. Kostman, I. Frank, M. Paiardini, D. Hazuda, G. Reyes-Terán, D. Richman, B. Howell, P. Tebas, J. Martinez-Picado, V. Planelles, M.J. Buzon, M.R. Betts, L.J. Montaner, CD32 is expressed on cells with transcriptionally active HIV but does not enrich for HIV DNA in resting T cells, Sci. Transl. Med. 10 (2018) eaar6759, , https://doi.org/10.1126/scitranslmed. aar6759.

[104] R. Badia, E. Ballana, M. Castellví, E. García-Vidal, M. Pujantell, B. Clotet, J.G. Prado, J. Puig, M.A. Martínez, E. Riveira-Muñoz, J.A. Esté, CD32 expression is associated to T-cell activation and is not a marker of the HIV-1 reservoir, Nat. Commun. 9 (2018) 2739, https://doi.org/10.1038/s41467-018-05157-w.

[105] G. Darcis, N. Kootstra, B. Hooibrink, T. van Montfort, K. Groen, S. Jurriaans, M. Bakker, C. Van Lint, B. Berkhout, A.O. Pasternak, CD32+CD4 + T cells are enriched in HIV-1 DNA, Transcription and Assembly (Frontiers in Retrovirology Conference 2018), KU, Leuven, Flanders, Belgium, 2018, pp. 43-44. 